\title{
Nonlinear stratified spindown over a slope
}

\author{
Jessica A. Benthuysen ${ }^{1,2,3, \dagger}$ and Leif N. Thomas ${ }^{4}$ \\ ${ }^{1}$ MIT/WHOI Joint Program, Woods Hole, MA 02543, USA \\ ${ }^{2}$ CSIRO Marine and Atmospheric Research, Hobart, Tasmania, 7000, Australia \\ ${ }^{3}$ Centre for Australian Weather and Climate Research, Hobart, Tasmania, 7000, Australia \\ ${ }^{4}$ Department of Environmental Earth System Science, Stanford University, Stanford, CA 94305, USA
}

(Received 3 August 2012; revised 25 February 2013; accepted 1 May 2013;

first published online 5 June 2013)

Nonlinear stratified spindown of an along-isobath current over an insulated slope is shown to develop asymmetries in the vertical circulation and vertical relative vorticity field. During spindown, cyclonic vorticity is weakened to a greater extent than anticyclonic vorticity near the boundary because of buoyancy advection. As a consequence, Ekman pumping is weakened over Ekman suction. Momentum advection can weaken Ekman pumping and strengthen Ekman suction. Time-dependent feedback between the geostrophic flow and the frictional secondary circulation induces asymmetry in cyclonic and anticyclonic vorticity away from the boundary. Buoyancy advection over a slope can modify the secondary circulation such that anticyclonic vorticity decays faster than cyclonic vorticity outside the boundary layer. In contrast, momentum advection can cause cyclonic vorticity to spin down faster than anticyclonic vorticity. A scaling and analytical solutions are derived for when buoyancy advection over a slope can have a more significant impact than momentum advection on these asymmetries. In order to test this scaling and analytical solutions, numerical experiments are run in which both buoyancy and momentum advection are active. These solutions are contrasted with homogeneous or stratified spindown over a flat bottom, in which momentum advection controls the asymmetries. These results are applied to ocean currents over continental shelves and slopes.

Key words: geophysical flows, ocean circulation, topographic effects

\section{Introduction}

When a geostrophic current flows over a boundary, frictional processes drive Ekman flows that have important consequences for the dynamics of the circulation as well as the modification and redistribution of tracers. Lateral variations in the Ekman transport induce Ekman pumping and suction that drive interior secondary circulations. On a flat bottom in a homogeneous fluid, classical spindown occurs when the frictionally driven secondary circulation decelerates the current. In linear theory, Ekman pumping is proportional to the vertical relative vorticity, $\zeta$ (Charney \& Eliassen 1949). In a homogeneous fluid, previous studies have shown that momentum advection causes asymmetry in the vertical velocity and vertical relative vorticity field. At order Rossby

$\dagger$ Email address for correspondence: jessica.benthuysen@csiro.au 
number, $\epsilon=U / f L$, Ekman advection of momentum weakens Ekman pumping in cyclonic regions, $\zeta>0$, and strengthens Ekman suction in anticyclonic regions, $\zeta<0$ (Benton, Lipps \& Tuann 1964; Hart 1995, 2000; Pedlosky 2008), where $U$ is the characteristic flow speed, $f$ is the planetary vorticity (assumed positive in this work), and $L$ is the characteristic lateral length scale.

Despite weaker Ekman pumping than Ekman suction, the frictionally driven circulation causes cyclonic vorticity to spin down faster than anticyclonic vorticity because of lateral momentum advection (Zavala Sansón \& van Heijst 2000; Zavala Sansón 2001; Benthuysen \& Thomas 2012a). These aspects of nonlinear spindown are complicated in a stratified fluid, in which diffusion and buoyancy advection are active. Furthermore, over a slope, the frictionally driven circulation couples with the buoyancy field. The purpose of this work is to show how buoyancy advection can modify the asymmetry in Ekman pumping and suction as well as anticyclonic and cyclonic vorticity during stratified spindown over a slope.

\subsection{Stratified spindown over a flat bottom}

Over a flat bottom in a semi-infinite vertical domain, the stratified, geostrophic flow adjusts in three stages in the limit $\epsilon \ll 1$. First, within an inertial period, $\mathscr{T}_{\text {inertial }}=2 \pi f^{-1}$, an Ekman layer forms in a depth $\delta_{e}=\sqrt{2 v / f}$, where $v$ is the kinematic viscosity. Then, Ekman pumping and suction sets up an interior secondary circulation that decelerates the geostrophic flow. Stratification limits the vertical penetration of the secondary circulation over a Prandtl depth, $H_{P}=f L / N$ (e.g. Holton $1965)$, where $N$ is the buoyancy frequency. The geostrophic flow decelerates on a spindown time scale, $\mathscr{T}_{\text {spindown }}=E^{-1 / 2} f^{-1}$, where the Ekman number, $E=\left(\delta_{e} / H_{P}\right)^{2}$, is assumed small. For an insulated boundary, Holton (1965) also showed that density variations occur over a diffusive boundary layer of depth $\delta_{T}=E^{1 / 4} H_{P}$, which is thicker than the Ekman layer. We refer to this diffusive boundary layer as the thermal boundary layer. For initially constant stratification, the buoyancy field adjacent to the boundary evolves uniformly by diffusion and remains decoupled from the Ekman dynamics in the small-Rossby-number regime. Viscous effects arise in the interior flow on a diffusive time scale, $\mathscr{T}_{\text {diffusive }}=E^{-1} f^{-1}$, and remove the geostrophic shear left by spindown.

In the interior, cyclonic vorticity decays faster than anticyclonic vorticity for increasing Rossby number, in part due to lateral momentum advection. Since the interior geostrophic flow is vertically sheared, vertical momentum advection also contributes to this asymmetry. In regions of cyclonic vorticity, vertical advection brings lower-momentum fluid upward and tends to enhance spindown of the cyclone. In contrast, in regions of anticyclonic vorticity, vertical advection brings highermomentum fluid downward and tends to slow spindown of the anticyclone. Thus, during stratified spindown over a flat bottom, cyclones decay faster than anticyclones due to lateral and vertical momentum advection despite weaker Ekman pumping than Ekman suction.

\subsection{Stratified spindown over a slope}

Over an insulated slope inclined at an angle $\theta$ to the horizontal, the buoyancy field couples with the frictionally driven circulation and can suppress spindown. MacCready \& Rhines (1991) show that for a uniform flow, cross-isobath Ekman advection of density tilts the isopycnals over a diffusive boundary layer. This isopycnal tilt weakens the geostrophic flow overlying the Ekman layer, hence reducing the bottom stress. This process is described as buoyancy shutdown of the Ekman transport (MacCready 
\& Rhines 1991). For long times, the Ekman transport decays as $\left(t / \mathscr{T}_{\text {shutdown, } M R}\right)^{-1 / 2}$ (MacCready \& Rhines 1991), where $t$ is time, and approaches an upslope transport $M_{\text {Thorpe }}=\kappa \cot \theta$ (Thorpe 1987), where $\kappa$ is the diffusivity. The decay time scale is

$$
\mathscr{T}_{\text {shutdown,MR }}=\left(\sigma^{-1}+S\right)\left(\cos \theta S^{2}(1+S)\right)^{-1} f^{-1},
$$

where $\sigma=v / \kappa$ is the Prandtl number and $S=(N \tan \theta / f)^{2}$ is the slope Burger number (MacCready \& Rhines 1991).

When the initial flow has vertical relative vorticity, stratified spindown by Ekman pumping and suction also leads to a decaying Ekman transport in time. Then, both spindown and buoyancy shutdown couple in their influence on the Ekman transport (e.g. Chapman 2002). For small Rossby numbers and sufficiently small slope Burger numbers, buoyancy shutdown locally weakens the geostrophic vertical relative vorticity (Benthuysen 2010). Hence both Ekman pumping and Ekman suction are suppressed to the same extent.

In this work, we examine nonlinear stratified spindown over a slope and the ensuing asymmetries that arise in Ekman pumping and suction and cyclonic and anticyclonic vorticity. The spindown problem is formulated in $\S 2$. In $\S 3$, we determine a scaling for when buoyancy advection over a slope affects these asymmetries. In $\S 4$, a system of equations is used to solve for the nonlinear correction to stratified spindown of a sinusoidal laterally sheared current over a slope. The asymmetries arising from buoyancy advection over a slope are compared with nonlinear spindown over a flat bottom for both homogeneous and stratified fluids. In $\S 5$, numerical experiments are performed to examine the extent to which the analytical solutions are valid for idealized flows applicable to continental slopes. These numerical experiments support the scaling arguments and demonstrate that buoyancy advection can have a more significant impact than momentum advection on the asymmetries in the vertical velocity and vertical relative vorticity field. The numerical discretization of the system of equations is included in the Appendix. In $\S 6$, results are discussed and summarized.

\section{Formulation}

\subsection{Basic equations}

The adjustment of an along-isobath current is examined in an incompressible, Boussinesq fluid rotating about the vertical axis at an angular velocity $f / 2$. The coordinate system is rotated at an angle $\theta$ with respect to the horizontal, as shown in figure $2(a)$. The slope angle is assumed sufficiently small such that $\cos \theta \approx 1$ and $\sin \theta \approx \theta$. The density field is assumed only temperature-dependent and is defined in the unrotated coordinate system as $\rho=\rho_{o}+\hat{\rho}(\hat{z})-\left(\rho_{o} / g\right) b$, where $\hat{z}$ is the vertical coordinate and the background stratification is constant, $N^{2}=-\left(g / \rho_{o}\right) \mathrm{d} \hat{\rho} / \mathrm{d} \hat{z}$. Buoyancy, $b$, is defined as the buoyancy anomaly with respect to the background density field, $\rho_{o}+\hat{\rho}$. The total pressure field is decomposed into a component due to the background stratification and a dynamical component, $p$.

In the rotated coordinate frame, where $z$ is the coordinate normal to the topography, the flow is composed of an along-isobath current, $u$, in the $x$-direction, a cross-isobath flow, $v$, in the $y$-direction, and a flow normal to the sloping boundary, $w$, in the $z$-direction. The kinematic viscosity and the diffusivity are assumed constant, and the Prandtl number, $v / \kappa$, is assumed equal to one. No along-isobath variations are assumed. A semi-infinite vertical domain is considered, where the Prandtl depth $H_{P}$ is 
the current's characteristic vertical scale. The equations that describe the dynamics are

$$
\begin{gathered}
\frac{\partial u}{\partial t}+v \frac{\partial u}{\partial y}+w \frac{\partial u}{\partial z}-f(v+\theta w)=v\left(\frac{\partial^{2} u}{\partial y^{2}}+\frac{\partial^{2} u}{\partial z^{2}}\right) \\
\frac{\partial v}{\partial t}+v \frac{\partial v}{\partial y}+w \frac{\partial v}{\partial z}+f u=-\frac{1}{\rho_{o}} \frac{\partial p}{\partial y}-\theta b+v\left(\frac{\partial^{2} v}{\partial y^{2}}+\frac{\partial^{2} v}{\partial z^{2}}\right) \\
\frac{\partial w}{\partial t}+v \frac{\partial w}{\partial y}+w \frac{\partial w}{\partial z}+f \theta u=-\frac{1}{\rho_{o}} \frac{\partial p}{\partial z}+b+v\left(\frac{\partial^{2} w}{\partial y^{2}}+\frac{\partial^{2} w}{\partial z^{2}}\right) \\
\frac{\partial b}{\partial t}+v\left(\frac{\partial b}{\partial y}-N^{2} \theta\right)+w\left(\frac{\partial b}{\partial z}+N^{2}\right)=\kappa\left(\frac{\partial^{2} b}{\partial y^{2}}+\frac{\partial^{2} b}{\partial z^{2}}\right) \\
\frac{\partial v}{\partial y}+\frac{\partial w}{\partial z}=0
\end{gathered}
$$

The equations are non-dimensionalized as follows (primes indicate dimensionless variables):

$$
\begin{gathered}
y=L y^{\prime}, \quad z=H_{P} z^{\prime}, \quad t=\mathscr{T}_{\text {spindown }} t^{\prime}, \\
u=U u^{\prime}, \quad v=U v^{\prime}, \quad w=U \Gamma w^{\prime}, \\
b=N^{2} H_{P} b^{\prime}, \quad p=\rho_{o} N^{2} H_{P}^{2} p^{\prime},
\end{gathered}
$$

where $U$ is a characteristic along-isobath flow speed, $L$ is a characteristic lateral length scale, and $\Gamma=H_{P} / L$ is the aspect ratio, which is equal to the Prandtl ratio, $f / N$.

The dimensionless equations (primes dropped) are

$$
\begin{gathered}
E^{1 / 2} \frac{\partial u}{\partial t}+\epsilon\left(v \frac{\partial u}{\partial y}+w \frac{\partial u}{\partial z}\right)-(v+\theta \Gamma w)=\frac{E}{2}\left(\Gamma^{2} \frac{\partial^{2} u}{\partial y^{2}}+\frac{\partial^{2} u}{\partial z^{2}}\right) \\
E^{1 / 2} \frac{\partial v}{\partial t}+\epsilon\left(v \frac{\partial v}{\partial y}+w \frac{\partial v}{\partial z}\right)+u=-\epsilon^{-1} \frac{\partial p}{\partial y}-\epsilon^{-1} S^{1 / 2} b+\frac{E}{2}\left(\Gamma^{2} \frac{\partial^{2} v}{\partial y^{2}}+\frac{\partial^{2} v}{\partial z^{2}}\right) \\
\epsilon \Gamma E^{1 / 2} \frac{\partial w}{\partial t}+\epsilon^{2} \Gamma^{2}\left(v \frac{\partial w}{\partial y}+w \frac{\partial w}{\partial z}\right)+\epsilon S^{1 / 2} \Gamma^{2} u \\
=-\frac{\partial p}{\partial z}+b+\frac{E}{2} \epsilon \Gamma^{2}\left(\Gamma^{2} \frac{\partial^{2} w}{\partial y^{2}}+\frac{\partial^{2} w}{\partial z^{2}}\right) \\
E^{1 / 2} \frac{\partial b}{\partial t}+\epsilon v\left(\frac{\partial b}{\partial y}-S^{1 / 2}\right)+\epsilon w\left(\frac{\partial b}{\partial z}+1\right)=\frac{E}{2}\left(\Gamma^{2} \frac{\partial^{2} b}{\partial y^{2}}+\frac{\partial^{2} b}{\partial z^{2}}\right) \\
\frac{\partial v}{\partial y}+\frac{\partial w}{\partial z}=0
\end{gathered}
$$

\subsection{Boundary and initial conditions}

In the rotated coordinate frame, the equations are solved subject to the following dimensionless no-slip, no-normal-flow, and insulated boundary conditions:

$$
\begin{gathered}
u=v=0 \quad \text { at } z=0, \\
w=0 \quad \text { at } z=0, \\
\frac{\partial b}{\partial z}+1=0 \quad \text { at } z=0 .
\end{gathered}
$$


The horizontal domain is assumed laterally unbounded. In the semi-infinite vertical domain,

$$
\begin{gathered}
u \rightarrow u(t=0, y) \quad \text { as } z \rightarrow \infty, \\
v, w, b \rightarrow 0 \quad \text { as } z \rightarrow \infty .
\end{gathered}
$$

The time-dependent adjustment problem is examined for an along-isobath flow with a sinusoidal lateral structure. In the rotated coordinate frame, the initial, dimensionless, leading-order flow is

$$
u(t=0, y)=\cos (y) .
$$

\subsection{Parameter space}

Nonlinear spindown over a slope is investigated when buoyancy shutdown can modify the leading-order dynamics. Other frictionally driven circulations can develop to modify the current's structure during spindown. For example, from the insulated boundary condition (2.16), diffusion of the initial stratification produces a laterally uniform buoyancy anomaly, $\bar{b}$, in the thermal boundary layer. Previous studies (e.g. Thorpe 1987; MacCready \& Rhines 1991; Benthuysen \& Thomas 2012b) have shown that this process drives an Ekman transport of magnitude $\kappa / \theta$ in steady state. When this steady-state transport is much less than the initial Ekman transport induced by the current, $\delta_{e} U / 2$, then the laterally uniform solution's effect on spindown is negligible. This condition, $\kappa / \theta \ll\left|\delta_{e} U / 2\right|$, will be considered here and written in terms of nondimensional parameters as $E^{1 / 2}<\epsilon S^{1 / 2}$.

Spindown is considered for small slope Burger numbers, $S \ll 1$, which is typical of mid-latitude continental shelves and slopes. A small aspect ratio is assumed, $\Gamma \ll 1$, so that the hydrostatic relation holds in (2.11). Following past stratified spindown theory, we consider the different dynamical balances to hold within an Ekman layer, a diffusive thermal boundary layer, and a far-field interior region. As described in $\S 1.1$, the thermal boundary layer depth is thicker than the Ekman layer depth for times longer than an inertial period. A scale separation exists between flows in the thermal boundary layer and the interior domain when $t<E^{-1} f^{-1}$, i.e. times that are less than a diffusive time scale. An example of the analytical solution with this boundary layer technique is shown in figure 1. This example illustrates that the cross-isobath flow varies over an Ekman layer depth, whereas the buoyancy field varies over a thicker thermal boundary layer depth. The along-isobath flow has significant vertical shear within the Ekman and thermal boundary layers and approaches its far-field state over a Prandtl depth.

Under the above conditions, the extent to which buoyancy shutdown reduces Ekman pumping during spindown is given by the parameter

$$
\beta \equiv \frac{\mathscr{T}_{\text {spindown }}}{\mathscr{T}_{\text {shutdown }}}=S^{2} E^{-1 / 2} .
$$

This key parameter measures the influence of buoyancy shutdown on stratified spindown and enters into the dynamics through the thermal boundary layer. The adjustment problem is examined for $\beta \leqslant 1$. We will present solutions with $\beta=0$, which corresponds to stratified spindown over a flat bottom, and $\beta=1$, in which Ekman advection of buoyancy modifies the leading-order Ekman flow. These $\beta$ values are chosen to illustrate the novel way in which buoyancy advection over a slope modifies the flow field. 
(a)

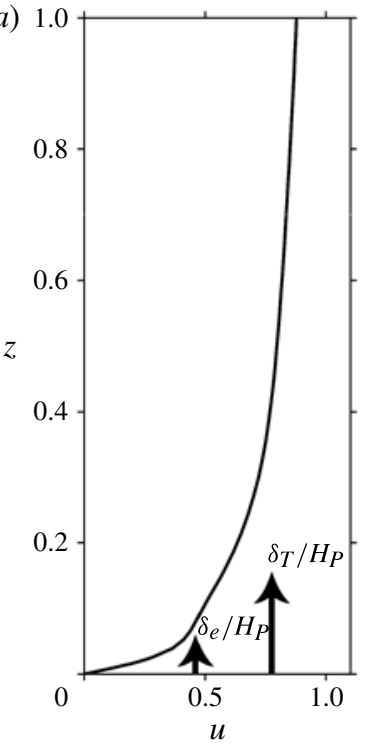

(b)

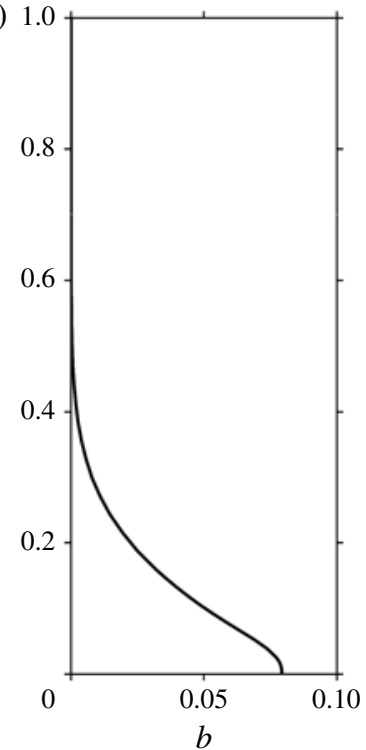

(c)

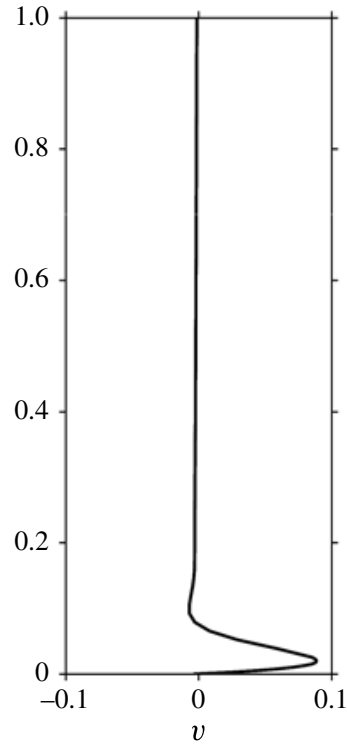

FIGURE 1. Example of the (a) along-isobath flow, $u,(b)$ buoyancy anomaly, $b$, and (c) cross-isobath flow, $v$, at $y=0$ from buoyancy shutdown solutions shown in figure 3 . Depth is non-dimensionalized by $H_{P}$ and the Ekman and thermal boundary layer depths are indicated. The interior domain is the region outside the boundary layers.

\section{Boundary layer decomposition and scaling arguments}

This section presents scaling arguments for the variables. Over a stratified slope, the Ekman flow couples with the buoyancy field. In the linear regime, the coupling symmetrically suppresses Ekman pumping and suction. This weakening slows the spindown of the geostrophic current. In this work, the linear analysis is extended into the nonlinear regime. During both homogeneous and stratified spindown over a flat bottom, momentum advection plays a dominant role in the asymmetry in Ekman pumping and suction and the asymmetrical spindown of cyclonic and anticyclonic vorticity. In contrast, over a stratified slope, buoyancy advection can dominate momentum advection in modifying the vertical velocity and vorticity fields. In this section, a scaling for the nonlinear correction to the flow field due to buoyancy advection is presented.

Following Thomas \& Rhines (2002), the variables are decomposed into contributions from the interior, Ekman layer and thermal boundary layer components, where the variables $u, v, w, p$ and $b$ are designated with the respective $i, e$, and $T$ subscripts. In the interior domain, a laterally sheared along-isobath flow evolves geostrophically from vortex stretching and squashing by an ageostrophic secondary circulation over a depth $H_{P}=f L / N$. In the Ekman layer, the momentum balance is between the Coriolis and frictional terms over a height $\delta_{e}=\sqrt{2 v / f}$, where the smallangle approximation is applied. In the thermal boundary layer, buoyancy variations occur over a diffusively growing depth $\sqrt{2 \kappa}$. The coordinate normal to the boundary is non-dimensionalized in the interior as $z^{\prime}=z / H_{P}$, in the thermal boundary layer as $\xi^{\prime}=z / \delta_{T}$, where $\delta_{T}=\left(2 \kappa \mathscr{T}_{\text {spindown }}\right)^{1 / 2}=E^{1 / 4} H_{P}$, and in the Ekman layer as $\eta^{\prime}=z / \delta_{e}$, where $\delta_{e}=E^{1 / 2} H_{P}$. 


\subsection{Stratified spindown: interior and Ekman layer scalings and equations}

In the interior, outside the viscous boundary layers, the along-isobath flow, $u_{i}=O(1)$, is hydrostatic and geostrophic. Hence, interior pressure scales as $p_{i}=O(\epsilon)$ from (2.10) and interior buoyancy scales as $b_{i}=O(\epsilon)$ from (2.11).

The interior along-isobath flow spins down through a frictionally driven ageostrophic circulation emanating from the top of the Ekman layer. At $O(1)$, the Ekman pumping condition is

$$
w_{i}(z=0)=-E^{1 / 2} \frac{1}{2} \frac{\partial u_{i}}{\partial y}(z=0),
$$

and $w_{i}, w_{e}=O\left(E^{1 / 2}\right)$. By continuity, the interior cross-isobath flow is $v_{i}=O\left(E^{1 / 2}\right)$. The secondary circulation $\psi$ is defined by $v=-\partial \psi / \partial z$ and $w=\partial \psi / \partial y$.

In order to satisfy no-slip boundary conditions, the Ekman layer velocity components are $u_{e}, v_{e}=O(1)$. From (2.12), cross-isobath Ekman advection of the background stratification balances buoyancy diffusion and $b_{e}=O\left(\epsilon S^{1 / 2}\right)$. From hydrostatic balance, $p_{e}=O\left(\epsilon S^{1 / 2} E^{1 / 2}\right)$.

\subsection{Buoyancy shutdown: thermal boundary layer scalings}

Linear stratified spindown is modified by Ekman advection of buoyancy over the slope. The process is illustrated in figure $2(a)$. By the insulating boundary condition (2.16), a thermal boundary layer develops through buoyancy diffusion, where a non-dimensional Ekman buoyancy flux $\partial b_{e} / \partial z \sim\left[b_{e}\right] /\left(\delta_{T} / H_{P}\right)$ is balanced by a thermal boundary layer buoyancy flux $\partial b_{T} / \partial z \sim\left[b_{T}\right] /\left(\delta_{T} / H_{P}\right)$, where [ ] denotes the variable's scale. Thus, the thermal boundary layer buoyancy anomaly scales as $b_{T}=O\left(\left[b_{e}\right]\left(\delta_{T} / \delta_{e}\right)\right)=O\left(\epsilon S^{1 / 2}\left(\delta_{T} / \delta_{e}\right)\right)$. From (2.11), pressure scales as $p_{T}=O\left(\left[b_{T}\right]\left(\delta_{T} / H_{P}\right)\right)=O\left(\epsilon S^{1 / 2}\left(\delta_{T} / \delta_{e}\right)\left(\delta_{T} / H_{P}\right)\right)$. From (2.10), the buoyancy anomaly balances a geostrophic along-isobath flow that scales as $u_{T}=O\left(\epsilon^{-1} S^{1 / 2}\left[b_{T}\right]\right)=$ $O\left(S\left(\delta_{T} / \delta_{e}\right)\right)$. By applying $\delta_{T} / \delta_{e}=E^{-1 / 4}$ and the $\beta$ parameter, the scaling for the along-isobath flow in the thermal boundary layer is $u_{T}=O\left(\beta^{1 / 2}\right)$. This flow opposes the interior along-isobath flow, reducing the geostrophic flow near the bottom. Hence, buoyancy shutdown suppresses the Ekman transport at $O\left(\beta^{1 / 2}\right)$ and causes symmetrical weakening in Ekman pumping and suction.

From (2.9), a cross-isobath flow develops at $v_{T}=O\left(\beta^{1 / 2} E^{1 / 2}\right)$. By continuity, the vertical velocity is $w_{T}=O\left(\beta^{1 / 2} E^{3 / 4}\right)$. This cross-isobath flow is weaker than the cross-isobath Ekman flow by $O\left(\beta^{1 / 2} E^{1 / 2}\right)$, and the vertical velocity is weaker than the interior and Ekman vertical velocity by $O\left(\beta^{1 / 2} E^{1 / 4}\right)$.

\subsection{Nonlinear buoyancy shutdown}

Next, we consider the scaling argument for when buoyancy advection leads to asymmetry during nonlinear spindown. Cross-isobath Ekman advection of the thermal boundary layer buoyancy anomaly in $\S 3.2$ leads to an additional buoyancy anomaly, $b_{e}^{\prime}$, in the Ekman layer. From (2.12), $\epsilon v_{e} \partial b_{T} / \partial y \sim \partial^{2} b_{e}^{\prime} / \partial \eta^{2}$ and $b_{e}^{\prime}=$ $O\left(\epsilon\left[b_{e}\right]\left(\delta_{T} / \delta_{e}\right)\right)=O\left(\epsilon^{2} S^{1 / 2}\left(\delta_{T} / \delta_{e}\right)\right)$. The Ekman flow advects buoyancy toward (away from) the cyclonic (anticyclonic) axis of the interior flow.

By the insulating boundary condition, (2.16), an additional buoyancy anomaly develops in the thermal boundary layer, where $b_{T}^{\prime}=O\left(\left[b_{e}^{\prime}\right]\left(\delta_{T} / \delta_{e}\right)\right)=O\left(\epsilon^{2} S^{1 / 2}\left(\delta_{T} / \delta_{e}\right)^{2}\right)$. From (2.10), the corresponding geostrophic along-isobath flow scales as $u_{T}^{\prime}=$ $O\left(\epsilon^{-1} S^{1 / 2}\left[b_{T}^{\prime}\right]\right)=O\left(S\left(\delta_{T} / \delta_{e}\right)^{2} \epsilon\right)$. The additional buoyancy anomaly causes greater (less) weakening of the geostrophic flow in cyclonic (anticyclonic) regions than in the linear case. This weakening leads to weaker cyclonic vorticity than anticyclonic vorticity 
(a)

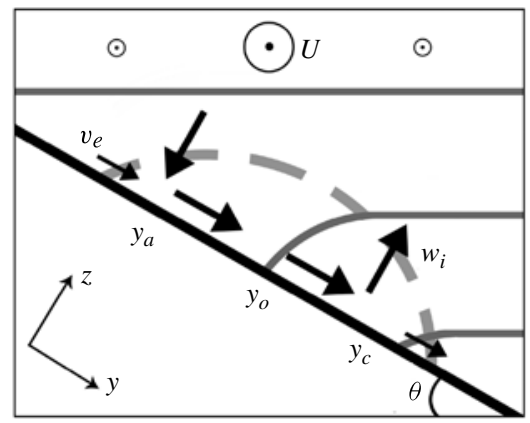

(b)

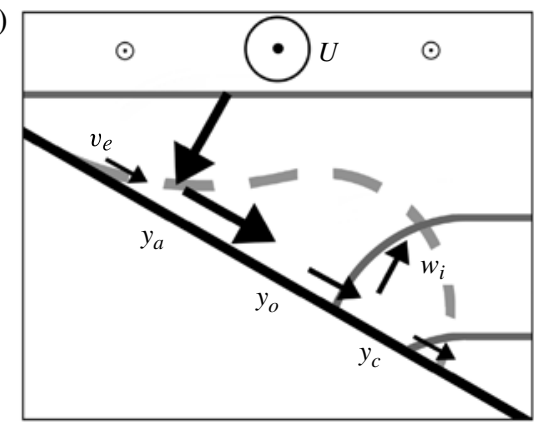

FIGURE 2. (a) Linear buoyancy shutdown. Consider the spindown of an initially barotropic jet, $U$, that is laterally symmetric about $y_{o}$. Convergence in the cross-isobath Ekman flow, $v_{e}$, induces Ekman pumping, $w_{i}(z=0)$, and suction. Since the jet is laterally symmetric, Ekman pumping on the cyclonic axis, $y_{c}$, is equal to Ekman suction on the anticyclonic axis, $y_{a}$. Ekman advection of the stratification leads to a positive buoyancy anomaly (enclosed within the grey dashed line) that is distributed over a thermal boundary layer depth. This positive buoyancy anomaly tilts the isopycnals (grey solid lines), weakening the bottom geostrophic velocity. Hence the Ekman flow is weakened, and Ekman pumping and suction are weakened to the same extent. (b) Nonlinear buoyancy shutdown. Consider the nonlinear adjustment due to stronger initial speed $U$. Two forms of buoyancy advection strengthen (weaken) the positive buoyancy anomaly (enclosed within the grey dashed line) around the cyclonic (anticyclonic) axis. First, cross-isobath Ekman flow, $v_{e}$, advects the buoyancy anomaly downslope. Second, in the thermal boundary layer, Ekman pumping (suction) advects the buoyancy anomaly upward (downward). The isopycnals (grey solid lines) tilt more steeply around the cyclonic axis. The resulting buoyancy anomaly enhances (reduces) the weakening of the Ekman flow around the cyclonic (anticyclonic) axis. Thus, Ekman pumping is suppressed to a greater extent than Ekman suction.

in the thermal boundary layer. From the Ekman pumping condition, (3.1), buoyancy advection weakens Ekman pumping to a greater extent than Ekman suction. We define this process as nonlinear buoyancy shutdown and it is illustrated in figure $2(b)$.

From the above scaling argument, the correction to the along-isobath flow by nonlinear buoyancy shutdown occurs at $O\left(S\left(\delta_{T} / \delta_{e}\right)^{2} \epsilon\right)=O\left(S E^{-1 / 2} \epsilon\right)$ and, in terms of $\beta$, is equal to

$$
\beta^{1 / 2} E^{-1 / 4} \epsilon \text {. }
$$

From the above scaling, nonlinear buoyancy shutdown can dominate momentum advection effects at $O(\epsilon)$ when $\beta>E^{1 / 2}$. Furthermore, nonlinear buoyancy shutdown enters into the leading-order dynamics when $\beta^{1 / 2} E^{-1 / 4} \epsilon>1$. In the following analysis, we examine how this mechanism affects the asymmetric spindown of cyclonic and anticyclonic vorticity.

In order to perform a perturbation expansion, the scaling (3.2) for the nonlinear correction is assumed less than $O(1)$. Furthermore, in order to illustrate the role of buoyancy advection during spindown over a slope, $\beta=O(1)$ is assumed. The Rossby number is constrained to $\epsilon \leqslant \beta^{-1 / 2} E^{1 / 4}$. From these assumptions, the dominant nonlinear correction for spindown over a stratified slope occurs by nonlinear buoyancy shutdown rather than momentum advection at $O(\epsilon)$.

From the above scaling arguments, the variables are expanded as

$$
u=u_{i}+\beta^{1 / 2} u_{T}+u_{e}
$$




$$
\begin{aligned}
v & =E^{1 / 2} v_{i}+\beta^{1 / 2} E^{1 / 2} v_{T}+v_{e}, \\
w & =E^{1 / 2} w_{i}+\beta^{1 / 2} E^{3 / 4} w_{T}+E^{1 / 2} w_{e}, \\
\psi & =E^{1 / 2} \psi_{i}+\beta^{1 / 2} E^{3 / 4} \psi_{T}+E^{1 / 2} \psi_{e}, \\
b & =\epsilon b_{i}+\beta^{1 / 2} S^{-1 / 2} \epsilon b_{T}+\epsilon S^{1 / 2} b_{e}, \\
p & =\epsilon p_{i}+S^{1 / 2} \epsilon p_{T}+\epsilon S^{1 / 2} E^{1 / 2} p_{e} .
\end{aligned}
$$

Next, a closed system of equations is formulated and solved to examine how nonlinear buoyancy shutdown affects Ekman pumping and suction and subsequently feeds back into the interior along-isobath flow.

\section{Analytical solutions}

\subsection{Expansion to $O\left(E^{-1 / 4} \epsilon\right)$}

In order to examine how nonlinear buoyancy shutdown affects the current's vertical circulation and spindown, the variables are expanded to $O\left(E^{-1 / 4} \epsilon\right)$ as follows:

$$
\begin{aligned}
(\boldsymbol{u}, b, p) & =\sum_{n=0}^{n=1}\left(\epsilon E^{-1 / 4}\right)^{n}\left(\boldsymbol{u}^{(n)}, b^{(n)}, p^{(n)}\right) \\
& =\left(\boldsymbol{u}^{(0)}, b^{(0)}, p^{(0)}\right)+\epsilon E^{-1 / 4}\left(\boldsymbol{u}^{(1)}, b^{(1)}, p^{(1)}\right) .
\end{aligned}
$$

At $n=0$, the vertical velocity and vertical vorticity decay symmetrically from buoyancy shutdown. The $n=1$ correction provides the asymmetric decay due to nonlinear buoyancy shutdown. The momentum and buoyancy equations are presented below to $O\left(\epsilon E^{-1 / 4}\right)$ for each region. The Ekman layer equations are

$$
\begin{gathered}
-v_{e}=\frac{1}{2} \frac{\partial^{2} u_{e}}{\partial \eta^{2}}, \\
u_{e}=\frac{1}{2} \frac{\partial^{2} v_{e}}{\partial \eta^{2}}, \\
-v_{e}+\epsilon E^{-1 / 4} v_{e} \frac{\partial b_{T}}{\partial y}(\xi=0)=\frac{1}{2} \frac{\partial^{2} b_{e}}{\partial \eta^{2}}, \\
\frac{\partial v_{e}}{\partial y}+\frac{\partial w_{e}}{\partial \eta}=0 .
\end{gathered}
$$

The thermal boundary layer variables are evaluated at the bottom owing to small vertical variations within the Ekman layer.

Next, the thermal boundary layer equations are

$$
\begin{gathered}
\frac{\partial u_{T}}{\partial t}-v_{T}+\epsilon E^{-1 / 4} w_{i}(z=0) \frac{\partial u_{T}}{\partial \xi}=\frac{1}{2} \frac{\partial^{2} u_{T}}{\partial \xi^{2}}, \\
u_{T}=-b_{T}, \\
\frac{\partial b_{T}}{\partial t}+\epsilon E^{-1 / 4} w_{i}(z=0) \frac{\partial b_{T}}{\partial \xi}=\frac{1}{2} \frac{\partial^{2} b_{T}}{\partial \xi^{2}}, \\
\frac{\partial v_{T}}{\partial y}+\frac{\partial w_{T}}{\partial \xi}=0 .
\end{gathered}
$$


In the thermal boundary layer, the interior vertical velocity is approximated by its value at the bottom owing to small vertical variations over the thermal boundary layer thickness.

The inviscid equations in the interior domain are

$$
\begin{gathered}
\frac{\partial u_{i}}{\partial t}-v_{i}=0, \\
u_{i}=-\frac{\partial p_{i}}{\partial y}, \\
0=-\frac{\partial p_{i}}{\partial z}+b_{i}, \\
\frac{\partial b_{i}}{\partial t}+w_{i}=0, \\
\frac{\partial v_{i}}{\partial y}+\frac{\partial w_{i}}{\partial z}=0 .
\end{gathered}
$$

The interior secondary circulation, $\psi_{i}$, is defined as $v_{i}=-\partial \psi_{i} / \partial z$ and $w_{i}=\partial \psi_{i} / \partial y$, where

$$
\frac{\partial^{2} \psi_{i}}{\partial y^{2}}+\frac{\partial^{2} \psi_{i}}{\partial z^{2}}=0
$$

is forced by Ekman pumping and suction at the boundary.

The equations are solved subject to the following boundary conditions:

$$
\begin{gathered}
u_{i}(z=0)+\beta^{1 / 2} u_{T}(\xi=0)+u_{e}(\eta=0)=0, \\
v_{e}(\eta=0)=0, \\
w_{i}(z=0)+w_{e}(\eta=0)=0, \\
\frac{\partial b_{T}}{\partial \xi}(\xi=0)+\frac{\partial b_{e}}{\partial \eta}(\eta=0)=0, \\
u_{i} \rightarrow u_{i}(t=0, y) \quad \text { as } z \rightarrow \infty, \\
v_{i}, w_{i}, b_{i} \rightarrow 0 \quad \text { as } z \rightarrow \infty, \\
u_{T}, v_{T}, w_{T}, b_{T} \rightarrow 0 \quad \text { as } \xi \rightarrow \infty, \\
u_{e}, v_{e}, w_{e}, b_{e} \rightarrow 0 \quad \text { as } \eta \rightarrow \infty .
\end{gathered}
$$

In the insulated boundary condition above, (4.20), the laterally uniform solution for buoyancy, $\bar{b}$, has been decomposed from the total solution. This solution satisfies $\partial \bar{b} / \partial z+1=0$. As stated in $\S 2.3$, this contribution to the total solution has been removed in order to focus on the solution arising from the initial along-isobath flow. The general Ekman layer solution is

$$
\begin{aligned}
u_{e}^{(n)} & =-\left(u_{i}^{(n)}(z=0)+\beta^{1 / 2} u_{T}^{(n)}(\xi=0)\right) \exp (-\eta) \cos \eta, \\
v_{e}^{(n)} & =\left(u_{i}^{(n)}(z=0)+\beta^{1 / 2} u_{T}^{(n)}(\xi=0)\right) \exp (-\eta) \sin \eta, \\
w_{e}^{(n)} & =\frac{1}{2} \frac{\partial}{\partial y}\left(u_{i}^{(n)}(z=0)+\beta^{1 / 2} u_{T}^{(n)}(\xi=0)\right) \exp (-\eta)(\sin \eta+\cos \eta),
\end{aligned}
$$


for $n=0,1$. The Ekman buoyancy solutions are

$$
\begin{aligned}
& b_{e}^{(0)}=-\left(u_{i}^{(0)}(z=0)+\beta^{1 / 2} u_{T}^{(0)}(\xi=0)\right) \exp (-\eta) \cos \eta \\
& b_{e}^{(1)}=-\left(u_{i}^{(1)}(z=0)+\beta^{1 / 2} u_{T}^{(1)}(\xi=0)+\varphi^{(0)} U_{T}^{(0)}(\xi=0) \sin (2 y)\right) \exp (-\eta) \cos \eta
\end{aligned}
$$

Next, the Ekman transport is defined as

$$
M_{e}=\int_{0}^{\infty} v_{e}(t, y, \eta) \mathrm{d} \eta=\sum_{n=0}^{n=1}\left(\epsilon E^{-1 / 4}\right)^{n} M_{e}^{(n)}
$$

and

$$
M_{e}^{(n)}=\frac{1}{2}\left(u_{i}^{(n)}(z=0)+\beta^{1 / 2} u_{T}^{(n)}(\xi=0)\right) .
$$

By the no-normal-flow boundary condition, Ekman pumping is given by

$$
w_{i}(z=0)=\sum_{n=0}^{n=1}\left(\epsilon E^{-1 / 4}\right)^{n} w_{i}^{(n)}(z=0)
$$

and

$$
w_{i}^{(n)}(z=0)=-\frac{1}{2} \frac{\partial}{\partial y}\left(u_{i}^{(n)}(z=0)+\beta^{1 / 2} u_{T}^{(n)}(\xi=0)\right) .
$$

The Ekman pumping solution is transformed into a boundary condition on $\psi_{i}$,

$$
\frac{\partial \psi_{i}^{(n)}}{\partial t}(z=0)=\frac{1}{2}\left(\frac{\partial \psi_{i}^{(n)}}{\partial z}(z=0)-\beta^{1 / 2} \frac{\partial u_{T}^{(n)}}{\partial t}(\xi=0)\right) .
$$

Next, the Ekman buoyancy solutions, (4.28) and (4.29), as well as the geostrophic relationship, (4.8), are used to transform the no-normal-buoyancy-flux boundary condition, (4.20), into a boundary condition on $u_{T}$,

$$
\frac{\partial u_{T}^{(n)}}{\partial \xi}(\xi=0)=u_{i}^{(n)}(z=0)+\beta^{1 / 2} u_{T}^{(n)}(\xi=0) .
$$

\section{2. $O(1)$ solution}

The $n=0$ solution is decomposed as

$$
\left(\psi_{i}^{(0)}, u_{i}^{(0)}, u_{T}^{(0)}\right)=\left(\varphi^{(0)}(t) \exp (-z), U_{i}^{(0)}(t, z), U_{T}^{(0)}(t, \xi)\right) \cos (y),
$$

where $\varphi^{(0)}$ measures the strength of the interior secondary circulation, which exponentially decays over the Prandtl depth. A closed set of partial differential equations is formed which couple the dynamics of both spindown and buoyancy shutdown, where

$$
\begin{aligned}
\frac{\partial U_{i}^{(0)}}{\partial t}-\varphi^{(0)}(t) \exp (-z) & =0, \\
\frac{\partial U_{T}^{(0)}}{\partial t}-\frac{1}{2} \frac{\partial^{2} U_{T}^{(0)}}{\partial \xi^{2}} & =0,
\end{aligned}
$$


subject to the boundary conditions

$$
\begin{gathered}
\frac{\mathrm{d} \varphi^{(0)}}{\mathrm{d} t}+\frac{1}{2}\left(\varphi^{(0)}+\beta^{1 / 2} \frac{\partial U_{T}^{(0)}}{\partial t}(\xi=0)\right)=0, \\
\frac{\partial U_{T}^{(0)}}{\partial \xi}(\xi=0)-U_{i}^{(0)}(z=0)-\beta^{1 / 2} U_{T}^{(0)}(\xi=0)=0, \\
U_{i}^{(0)} \rightarrow 1 \quad \text { as } z \rightarrow \infty, \\
U_{T}^{(0)} \rightarrow 0 \quad \text { as } \xi \rightarrow \infty,
\end{gathered}
$$

and the initial conditions

$$
\begin{aligned}
& \varphi^{(0)}(t=0)=-\frac{1}{2}, \\
& U_{i}^{(0)}(t=0)=1, \\
& U_{T}^{(0)}(t=0)=0 .
\end{aligned}
$$

This system of equations is solved using Laplace transforms in time, such that

$$
\begin{aligned}
\left(\widetilde{\varphi}(s), \widetilde{U}_{i}(s, z), \widetilde{U}_{T}(s, \xi)\right) & =\mathscr{L}\left[\varphi^{(0)}(t), U_{i}^{(0)}(t, z), U_{T}^{(0)}(t, \xi)\right] \\
& \equiv \int_{0}^{\infty}\left(\varphi^{(0)}(t), U_{i}^{(0)}(t, z), U_{T}^{(0)}(t, \xi)\right) \exp (-s t) \mathrm{d} t
\end{aligned}
$$

and

$$
\begin{aligned}
\widetilde{\varphi} & =-\frac{1}{1+2 s+\sqrt{2 \beta s}}, \\
\widetilde{U}_{i} & =\frac{1}{s}\left(1-\frac{\exp (-z)}{1+2 s+\sqrt{2 \beta s}}\right), \\
\widetilde{U}_{T} & =\frac{-2 \exp (-\xi \sqrt{2 s})}{2 \sqrt{\beta} s+(1+2 s) \sqrt{2 s}} .
\end{aligned}
$$

The solutions are determined by operating on (4.48)-(4.50) by the inverse Laplace transform (Abramowitz \& Stegun 1972). The temporal structure of the interior secondary circulation is

$$
\begin{aligned}
\varphi^{(0)}(t)= & -\frac{1}{2} \exp (a t) \cos (b t)+\sqrt{\frac{\beta t}{2 \pi}}-\frac{1}{4 b}(1+2 a) \exp (a t) \sin (b t) \\
& +\sqrt{\frac{\beta}{2 \pi}} \exp (a t)\left\{\cos (b t) \int_{0}^{t} \tau^{1 / 2} \exp (-a \tau)\right. \\
& \times\left(2 a \cos (b \tau)+\left[b^{2}-\frac{a^{2}}{b}\right] \sin (b \tau)\right) \mathrm{d} \tau \\
& \left.+\sin (b t) \int_{0}^{t} \tau^{1 / 2} \exp (-a \tau)\left(\left[-b+\frac{a^{2}}{b}\right] \cos (b \tau)+2 a \sin (b \tau)\right) \mathrm{d} \tau\right\},
\end{aligned}
$$

where the coefficients are $a=-(2-\beta) / 4$, which is negative for $\beta \leqslant 1$, and $b= \pm \sqrt{\beta(1-\beta / 4)} / 2$. In the limit $\beta \rightarrow 0$, the coefficients approach $a=-1 / 2$ and $b=0$ and, from the first term in (4.51), the Ekman pumping approaches the spindown solution over a flat bottom, where $\varphi_{\text {spindown }}(t)=-(1 / 2) \exp (-t / 2)$. The second term 
represents the opposition to spindown by buoyancy forces and initially grows as $\sqrt{\beta t /(2 \pi)}$. The third and fourth terms in (4.51) modulate the long-time behaviour of the Ekman pumping. Buoyancy forces cause Ekman pumping to initially decay as $\varphi^{(0)}(t \ll 1)=-(1 / 2) \exp ((\beta / 4-1 / 2) t)+\sqrt{\beta t /(2 \pi)}$.

In the thermal boundary layer, the buoyancy anomaly is associated with a geostrophically balanced along-isobath flow,

$$
\begin{aligned}
U_{T}^{(0)}(t, \xi>0)= & \frac{\exp (a t)}{\sqrt{2 \pi}}\left\{\sin (b t) \int_{0}^{t} \frac{\exp \left(-a \tau-\xi^{2} /(2 \tau)\right)}{\sqrt{\tau}}\right. \\
& \times\left(\left[\frac{\xi \sqrt{\beta}}{2 b \tau}-\frac{\beta}{4 b}\right] \cos (b \tau)-\sin (b \tau)\right) \mathrm{d} \tau \\
& +\cos (b t) \int_{0}^{t} \frac{\exp \left(-a \tau-\xi^{2} /(2 \tau)\right)}{\sqrt{\tau}} \\
& \left.\times\left(\left[-\frac{\xi \sqrt{\beta}}{2 b \tau}+\frac{\beta}{4 b}\right] \sin (b \tau)-\cos (b \tau)\right) \mathrm{d} \tau\right\}
\end{aligned}
$$

and

$$
\begin{aligned}
U_{T}^{(0)}(t, \xi=0)= & \frac{\sqrt{\beta}}{2 b} \exp (a t) \sin (b t)-\sqrt{\frac{2 t}{\pi}}+\frac{\exp (a t)}{\sqrt{2 \pi}}\left\{\sin (b t) \int_{0}^{t} \tau^{1 / 2} \exp (-a \tau)\right. \\
& \times\left(\left[-\frac{a \beta}{2 b}+2 b\right] \cos (b \tau)-\left[\frac{\beta}{2}+2 a\right] \sin (b \tau)\right) \mathrm{d} \tau \\
& +\cos (b t) \int_{0}^{t} \tau^{1 / 2} \exp (-a \tau)\left(\left[\frac{a \beta}{2 b}-2 b\right] \sin (b \tau)\right. \\
& \left.\left.-\left[\frac{\beta}{2}+2 a\right] \cos (b \tau)\right) \mathrm{d} \tau\right\}
\end{aligned}
$$

An initial along-isobath flow in the positive $x$-direction induces a downwelling Ekman flow that gives rise to a positive buoyancy anomaly in the thermal boundary layer. This buoyancy anomaly corresponds to an along-isobath flow that opposes the interior flow and initially grows as $\sqrt{2 t / \pi}$.

By suppressing Ekman pumping and suction, buoyancy shutdown inhibits the spindown of the interior along-isobath flow. By inverse Laplace transform of (4.49), the interior along-isobath flow is

$$
\begin{aligned}
U_{i}^{(0)}(t, z)= & \{1-\exp (-z)(1-\cos (b t) \exp (a t))\}-\frac{\beta}{4 b} \exp (-z+a t) \sin (b t) \\
& +\frac{1}{b} \sqrt{\frac{\beta}{2 \pi}} \exp (-z+a t)\left\{\sin (b t) \int_{0}^{t} \tau^{1 / 2} \exp (-a \tau)(a \cos (b \tau)\right. \\
& \left.+b \sin (b \tau)) \mathrm{d} \tau+\cos (b \tau) \int_{0}^{t} \tau^{1 / 2} \exp (-a \tau)(-a \sin (b \tau)+b \cos (b \tau)) \mathrm{d} \tau\right\} .
\end{aligned}
$$

The first term, which is enclosed by braces, approaches the spindown solution in the limit $\beta \rightarrow 0$, where $U_{i, \text { spindown }}=1-\exp (-z)(1-\exp (-t / 2))$. For $\beta=1$, buoyancy shutdown reduces the rate at which the interior along-isobath flow decays, but the flow continues to decay in time because Ekman pumping remains non-zero. 

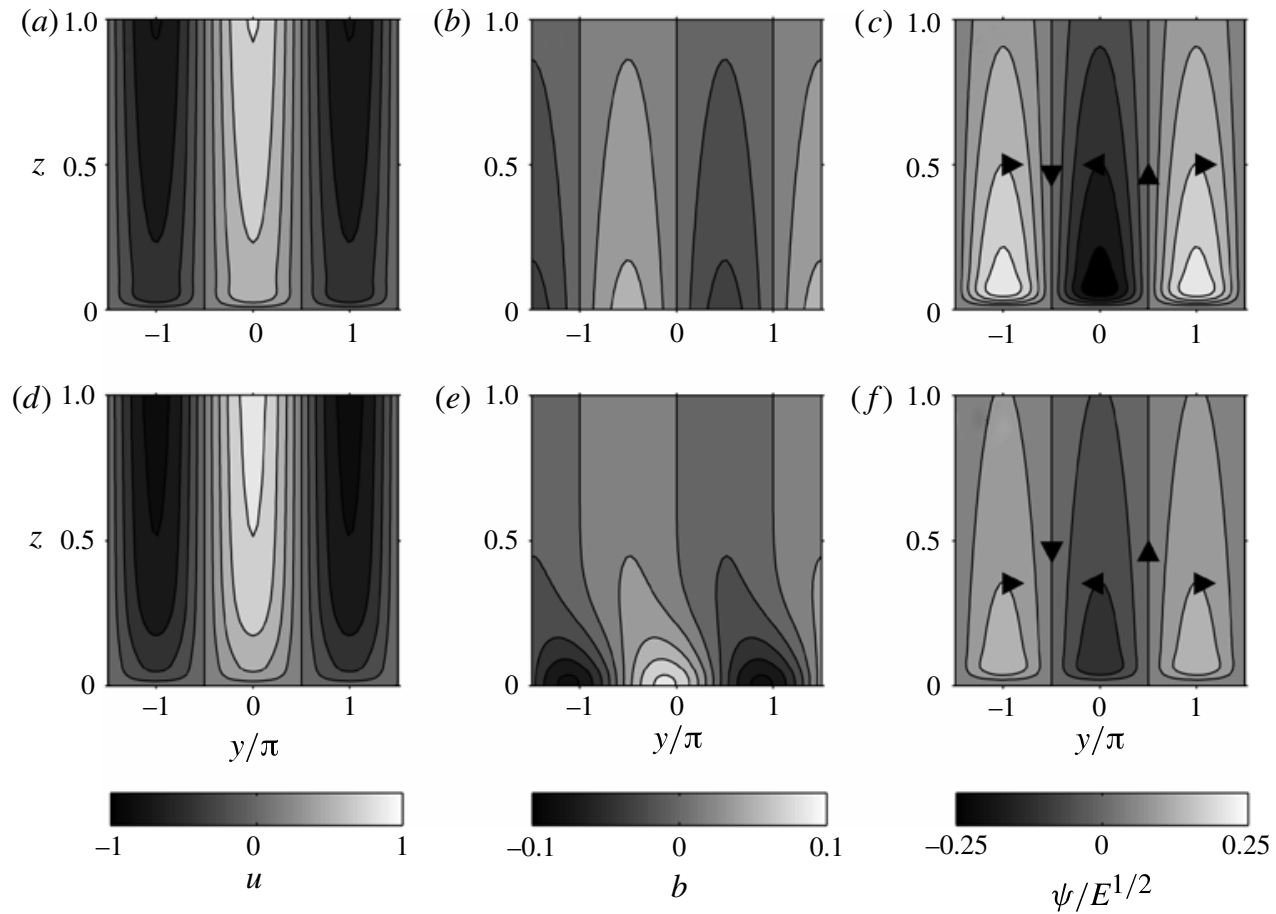

FIgURE 3. The $n=0$ analytical solutions for $\beta=0(a-c)$ and $\beta=1(d-f)$. The sections are shown at $t=1.4$. The along-isobath flow, $u(a, d)$, is contoured every 0.2 units, the buoyancy anomaly, $b(b, e)$, is contoured every 0.02 units, and the secondary circulation, $\psi / E^{1 / 2}(c, f)$, is contoured every 0.05 units. The arrows indicate the direction of the secondary circulation. The solutions are plotted with $E=6.42 \times 10^{-4}, \epsilon=0.094, S=0(a-c)$, and $S=0.16(d-f)$.

In figure 3 , the solutions are plotted for stratified spindown over a flat bottom, $\beta=0$, and over a slope, $\beta=1$. For $\beta=1$, the variables are mapped onto the rotated coordinate frame to compare with the flat bottom case. The flow is plotted at $t=1.4$. At this time, the Ekman pumping solution for $\beta=0$ is half of its initial value and the thermal boundary layer buoyancy anomaly $\beta=1$ is at its maximum value.

Over a flat bottom, $\beta=0$, the interior secondary circulation spins down the alongisobath flow. This circulation vertically advects the stratification and gives rise to negative (positive) buoyancy anomalies in regions of Ekman pumping (suction). Over a slope with $\beta=1$, positive and negative buoyancy anomalies form away from regions of Ekman pumping and suction. These buoyancy anomalies are due to cross-isobath Ekman advection of buoyancy and are the components of the buoyancy field that lead to buoyancy shutdown of the Ekman transport. For $\beta=1$, buoyancy shutdown is evident with a weaker secondary circulation and an along-isobath flow that is not as weak away from the boundary.

$$
\text { 4.3. } O\left(\beta^{1 / 2} E^{-1 / 4} \epsilon\right) \text { solution }
$$

The $n=1$ solution structure is decomposed as

$$
\left(\psi_{i}^{(1)}, u_{i}^{(1)}, u_{T}^{(1)}\right)=\left(\varphi^{(1)}(t) \exp (-2 z) \sin (2 y), U_{i}^{(1)}(t) \exp (-2 z) \sin (2 y), U_{T}^{(1)}(t, \xi) \sin (2 y)\right) .
$$


The lateral length scale of the second harmonic is half of the lowest-order mode. Subsequently, by (4.16), the interior secondary circulation is confined closer to the boundary over half of the Prandtl depth. The time evolution of the nonlinear correction is determined by the following closed set of partial differential equations:

$$
\begin{gathered}
\frac{\mathrm{d} U_{i}^{(1)}}{\mathrm{d} t}-2 \varphi^{(1)}=0 \\
\frac{\partial U_{T}^{(1)}}{\partial t}-\frac{1}{2} \frac{\partial^{2} U_{T}^{(1)}}{\partial \xi^{2}}=\frac{1}{2} \varphi^{(0)} \frac{\partial U^{(0)}}{\partial \xi},
\end{gathered}
$$

subject to the boundary conditions

$$
\begin{gathered}
\frac{\partial \varphi^{(1)}}{\partial t}+\left(\varphi^{(1)}+\frac{1}{2} \beta^{1 / 2} \frac{\partial U_{T}^{(1)}}{\partial t}(\xi=0)\right)=0 \\
\frac{\partial U_{T}^{(1)}}{\partial \xi}(\xi=0)-U_{i}^{(1)}-\beta^{1 / 2} U_{T}^{(1)}(\xi=0)=\varphi^{(0)} U_{T}^{(0)}(\xi=0), \\
U_{T}^{(1)} \rightarrow 0 \quad \text { as } \xi \rightarrow \infty
\end{gathered}
$$

and the intial condition

$$
\left(\varphi^{(1)}(t=0), U_{i}^{(1)}(t=0, z), U_{T}^{(1)}(t=0)\right)=(0,0,0) .
$$

This set of equations is solved numerically for $\beta=1$ by the Crank-Nicolson scheme. The details of the numerical calculation are provided in the Appendix. Next, the correction to the vertical velocity and vertical relative vorticity from nonlinear buoyancy shutdown is presented. These calculations are compared with the analytical $O(\epsilon)$ correction from momentum advection during homogeneous spindown over a flat bottom.

\subsection{Asymmetries during nonlinear buoyancy shutdown versus nonlinear homogeneous spindown over a flat bottom}

During nonlinear homogeneous spindown (HSD) over a flat bottom and nonlinear stratified spindown (SSD) over a slope, asymmetries arise in the vertical velocity and vertical vorticity field. For HSD over a flat bottom in a finite depth $H$, where $\Gamma=H / L$ and $E=\left(\delta_{e} / H\right)^{2}$, the interior vertical velocity and vertical relative vorticity are expanded in $\epsilon$ as

$$
\begin{gathered}
w_{i, H S D}=w_{i}^{(0)}+\epsilon w_{i}^{(1)}, \\
\zeta_{i, H S D}=\zeta_{i}^{(0)}+\epsilon \zeta_{i}^{(1)} .
\end{gathered}
$$

For an initial flow $u=\cos (y)$, the solutions to $O(\epsilon)$ are (Benthuysen \& Thomas 2012b)

$$
\begin{aligned}
\zeta_{i, H S D}^{(0)} & =\sin (y) \exp (-t / 2), \\
\zeta_{i, H S D}^{(1)} & =\frac{13}{20} \cos (2 y)(\exp (-t / 2)-\exp (-t)), \\
w_{i, H S D}^{(0)} & =\frac{1}{2} \sin (y) \exp (-t / 2)(1-z), \\
w_{i, H S D}^{(1)} & =\cos (2 y)\left(\frac{13}{40} \exp (-t / 2)-\frac{6}{40} \exp (-t)\right)(1-z) .
\end{aligned}
$$




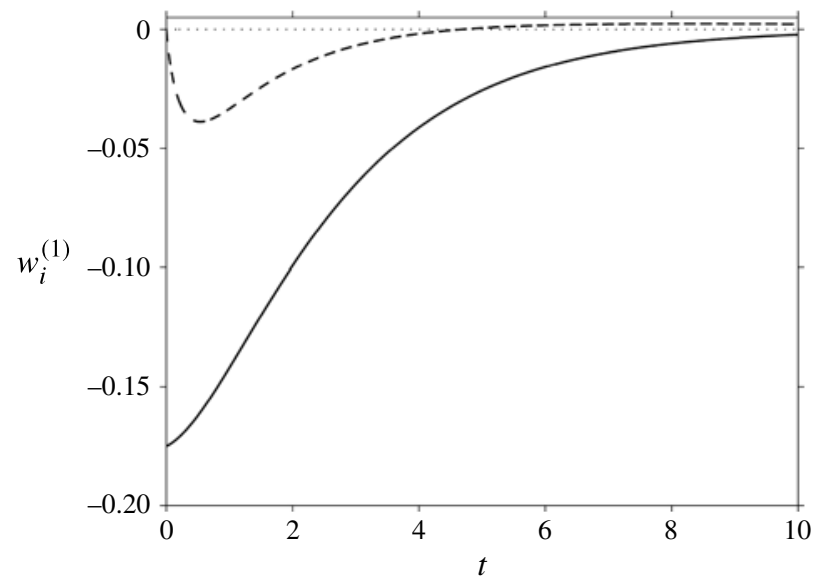

FIGURE 4. The analytical nonlinear correction to Ekman pumping and suction on the cyclonic and anticyclonic axis, respectively, is shown given $u(t=0)=\cos (y)$. These corrections are compared between HSD (solid line) and SSD over a slope (dashed line) with $\beta=1$. The axis $w_{i}^{(1)}(z=0)=0$ is indicated by the dotted line.

Figure 4 compares the nonlinear corrections to Ekman pumping on the cyclonic axis, $y_{c}=\pi / 2+2 m \pi$, and Ekman suction on the anticyclonic axis, $y_{a}=-\pi / 2+2 m \pi$, where $m$ is an integer. For nonlinear buoyancy shutdown, $w_{i}^{(1)}(z=0)=2 \varphi^{(1)} \cos (2 y)$ and for nonlinear homogeneous spindown, $w_{i}^{(1)}(z=0)$ is given by (4.67) evaluated at $z=0$. In both cases, the nonlinear corrections are both negative.

Although Ekman pumping is weaker than Ekman suction for both cases, their temporal evolution reveals significant differences. During HSD, Ekman and interior momentum advection contribute to the nonlinear correction. Initially, this correction is non-zero because the Ekman flow is set-up within an inertial period. Since the correction is negative, Ekman pumping is weakened at $y_{c}$ and Ekman suction is strengthened at $y_{a}$. However, during SSD over a slope, nonlinear buoyancy shutdown weakens Ekman pumping more than Ekman suction. This correction is initially zero and grows to its maximum negative value at $t=0.5$. Then, as the buoyancy anomaly diffuses away from the boundary and the geostrophic flow weakens, the correction decays in time. The correction reverses sign at $t=4.7$, reaches its maximum positive value at $t=8.3$, and finally decays to zero as the along-isobath flow spins down.

Despite weaker Ekman pumping than Ekman suction from either momentum or buoyancy advection, each mechanism causes different asymmetries in cyclonic and anticyclonic vorticity. The nonlinear correction to the vertical relative vorticity is shown in figure 5. During HSD, the nonlinear correction $\zeta_{i}^{(1)}$ at $y_{c}$ and $y_{a}$ from (4.65) is negative and corresponds to faster spindown of cyclonic vorticity than anticyclonic vorticity.

Similarly, buoyancy advection over a slope at $O\left(\beta^{1 / 2} E^{-1 / 4} \epsilon\right)$ can lead to asymmetric spindown. The geostrophic components of the vertical relative vorticity field are

$$
\begin{aligned}
& \zeta_{i}^{(1)}=-2 U_{i}^{(1)}(t) \exp (-2 z) \cos (2 y), \\
& \zeta_{T}^{(1)}=-2 U_{T}^{(1)}(t, \xi) \cos (2 y) .
\end{aligned}
$$




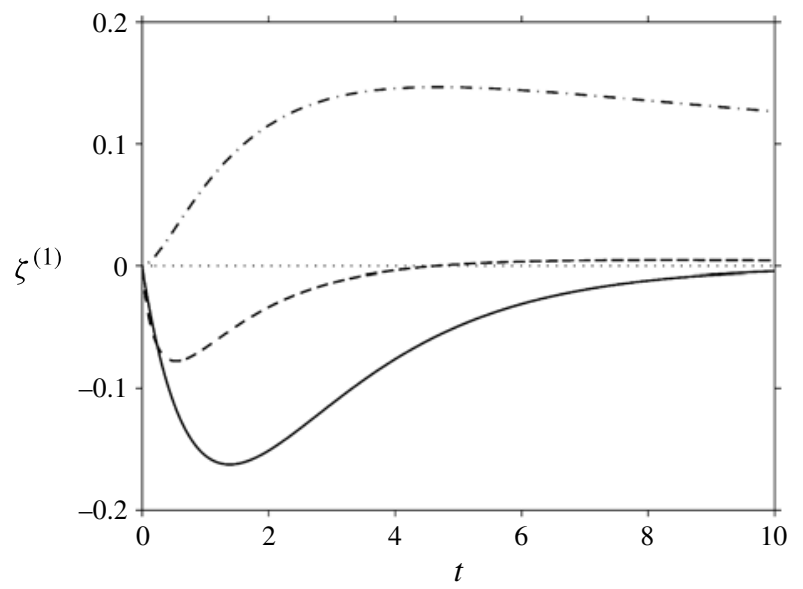

FIGURE 5. The analytical nonlinear correction to vertical relative vorticity on the cyclonic and anticyclonic axis. For HSD over a flat bottom, the correction is presented for the interior vertical relative vorticity, $\zeta_{i}^{(1)}$ (solid line). For SSD over a slope, with $\beta=1$, the corrections are presented for the interior component to the vertical relative vorticity evaluated at the bottom, $\zeta_{i}^{(1)}(z=0)$ (dot-dashed line), and total geostrophic component evaluated at the bottom, $\beta^{1 / 2} \zeta_{T}^{(1)}(\xi=0)+\zeta_{i}^{(1)}(z=0)$ (dashed line). The dotted line indicates $\zeta^{(1)}=0$.

The total nonlinear correction to vertical relative vorticity in the thermal boundary layer is measured by $\zeta_{i}^{(1)}(t, z=0)+\beta^{1 / 2} \zeta_{T}^{(1)}(t, \xi=0)$ evaluated on the cyclonic or anticyclonic axis. Figure 5 shows the analytical corrections to the vertical relative vorticity field in the interior and the thermal boundary layer. With $\beta=1$, the nonlinear correction is greater than $O(\epsilon)$ by $E^{-1 / 4}$. In the thermal boundary layer, nonlinear buoyancy shutdown initially weakens the geostrophic flow to a greater extent around the cyclonic axis than the anticyclonic axis. This weakening causes the cyclonic vorticity to decay faster than the anticyclonic vorticity in the thermal boundary layer and corresponds to a negative correction.

When nonlinear buoyancy shutdown suppresses Ekman pumping more than Ekman suction, the interior secondary circulation can cause cyclonic vorticity to initially decay more slowly than anticyclonic vorticity in the interior. The correction to the interior vertical relative vorticity, $\zeta_{i}^{(1)}(z=0)$, is positive and reaches a maximum at $t=4$.7. At this time, the correction to the interior secondary circulation changes sign and spins down the correction to the interior geostrophic flow.

This solution does not account for momentum advection in the interior. The homogeneous spindown solution shows that momentum advection preferentially spins down cyclonic vorticity at $O(\epsilon)$. This mechanism can counter nonlinear buoyancy shutdown's effect on the interior vorticity field. We will investigate these competing processes in the simulations.

In the next section, numerical simulations are run to address two questions. First, to what extent does the theory of nonlinear buoyancy shutdown explain the asymmetry in Ekman pumping and suction? Second, does the asymmetry in cyclonic and anticyclonic vorticity evolve as predicted by nonlinear buoyancy shutdown when momentum advection is also present? It will be shown that nonlinear buoyancy shutdown effects are necessary to interpret the asymmetries in the vertical velocity and vertical vorticity fields. 
Configuration

Homogeneous spindown (HSD)

Stratified spindown (SSD)

Stratified spindown (SSD)

$\begin{array}{cccc}\theta & N^{2}\left(\mathrm{~s}^{-2}\right) & H(\mathrm{~m}) & H_{P}(\mathrm{~m}) \\ 0 & 0 & 266 & - \\ 0 & 1.6 \times 10^{-5} & 4500 & 266 \\ 0.01 & 1.6 \times 10^{-5} & 4500 & 266\end{array}$

TABLE 1. ROMS model configurations.

\section{Numerical experiments}

\subsection{Numerical model set-up}

Numerical experiments are run to compare homogeneous spindown (HSD) over a flat bottom, stratified spindown (SSD) over a flat bottom, and stratified spindown over a slope. The model is run for increasing Rossby number within and outside the parameter range for which the analytical theory is valid. For stratified spindown over a slope, the experiments are designed to show that the asymmetry in Ekman pumping and suction cannot be explained by momentum advection alone.

Nonlinear spindown is investigated in a series of numerical experiments with application to mid-latitude currents over continental slopes. The experiments are performed using the regional ocean modelling system (ROMS) (Shchepetkin \& McWilliams 2005), which is a free-surface, hydrostatic, primitive-equation model with stretched, terrain-following coordinates. This model is configured with no alongisobath variations and a bottom boundary that is inclined to the horizontal at an angle $\theta$. The domain is $300 \mathrm{~km}$ wide with a uniform horizontal grid spacing of $1 \mathrm{~km}$ and is bounded by sidewalls.

Three model configurations are considered, as shown in table 1. For HSD over a flat bottom, the domain height is $H=266 \mathrm{~m}$ so that the spindown time is the same as in the stratified configurations. In this set-up, the vertical domain has 60 grid points, with a vertical grid resolution varying from 1 to $6 \mathrm{~m}$. In the stratified configurations, the maximum depth is $4.5 \mathrm{~km}$, with a variable vertical grid of 201 points and vertical grid resolution that ranges from 1 to $55 \mathrm{~m}$. At the bottom, density satisfies the insulated boundary condition and an approximate no-slip boundary condition is imposed. For comparison with the analytical theory, the mixing coefficients are uniform and equal to $v, \kappa=2.27 \times 10^{-3} \mathrm{~m}^{2} \mathrm{~s}^{-1}$ so that $\sigma=1$. Uniform rotation is applied with a midlatitude Coriolis parameter $f=10^{-4} \mathrm{~s}^{-1}$. The Ekman layer depth is $\delta_{e}=6.7 \mathrm{~m}$, and $E=6.42 \times 10^{-4}, \Gamma=0.025$.

The initial parameters are chosen within the range of current characteristics over the continental slope. The initial along-isobath flow is given by $u(t=0, y)=U \cos (y / L)$, where $U=0.1,0.2,0.3,0.4,0.5 \mathrm{~m} \mathrm{~s}^{-1}, L=10.6 \mathrm{~km}$ for a narrow slope current. For the stratified configurations, the initial stratification has a buoyancy frequency $N$, where $N^{2}=1.6 \times 10^{-5} \mathrm{~s}^{-2}$. From these parameters, the Prandtl depth is $H_{P}=266 \mathrm{~m}$. For the flat bottom configuration, $\theta=0$ and $\beta=0$, and for the continental slope configuration, $\theta=0.01, S=0.16$ and $\beta=1$. The spindown time scale is $\mathscr{T}_{\text {spindown }}=6.3$ inertial periods.

The model output is decomposed into components corresponding to laterally uniform and cross-isobath-varying flows. This decomposition was done to remove the laterally uniform contribution to the flow that arises from the adjustment of the uniform stratification. In order to isolate the laterally varying solution, the model is run with no initial flow, and this solution is subtracted from the total model output. This 

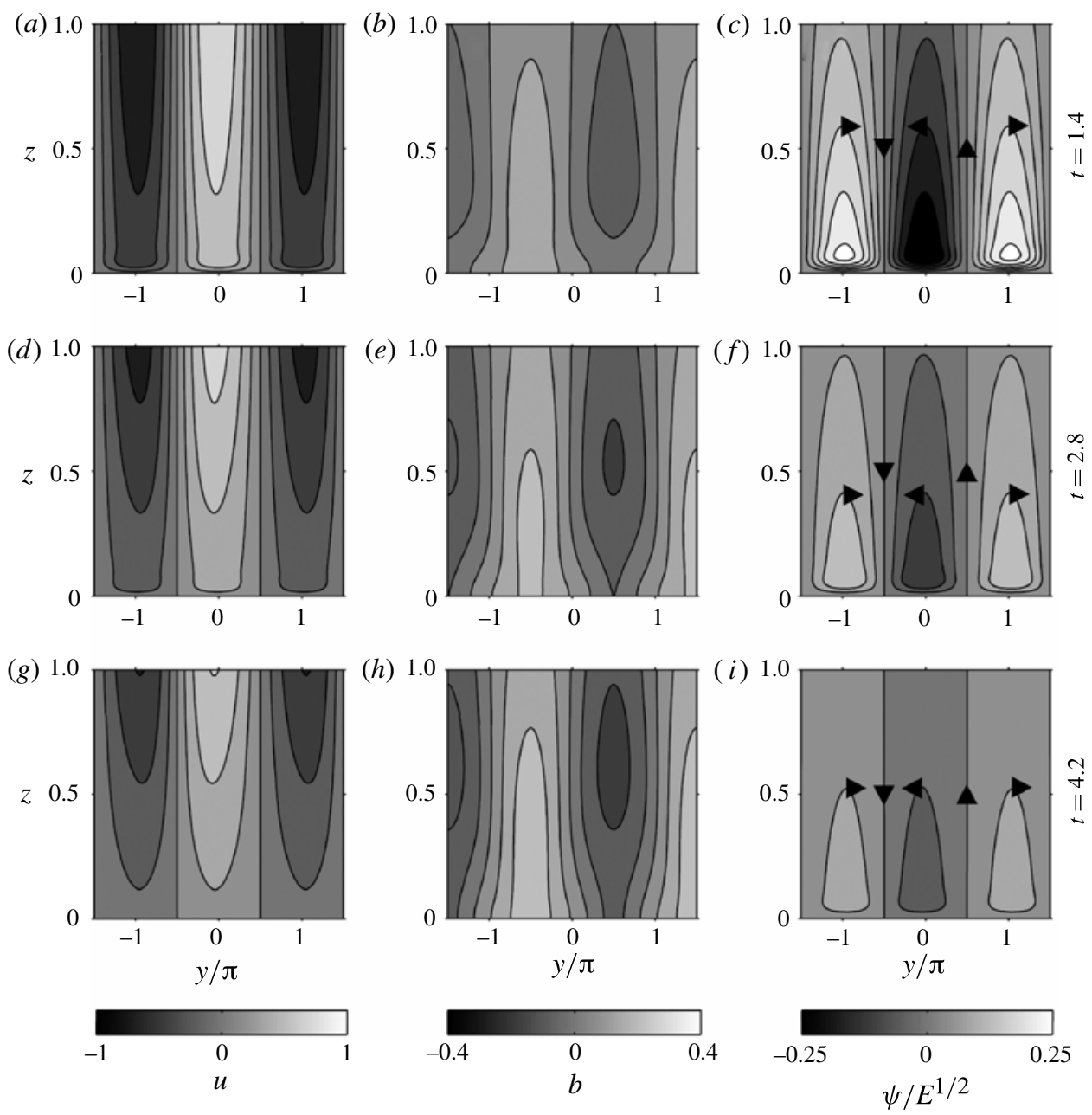

FIgURE 6. $\beta=0, \theta=0, \epsilon=0.4$. The stratified spindown ROMS solutions are shown at $t=1.4(a-c), 2.8(d-f)$, and $4.2(g-i)$. The along-isobath flow, $u(a, d, g)$, is contoured every 0.2 units, the buoyancy anomaly, $b(b, e, h)$, is contoured every 0.08 units, and the secondary circulation, $\psi / E^{1 / 2}(c, f, i)$, is contoured every 0.05 units.

decomposition assumes that the coupling between the laterally uniform and laterally sheared flow is a higher-order effect. In order to focus on the system's subinertial adjustment, the model output is further processed by filtering the secondary circulation by a Butterworth low-pass filter with a cut-off frequency of $0.12 f$.

\subsection{Asymmetries in Ekman pumping and suction}

In this section, the asymmetry in Ekman pumping and suction is examined for increasing Rossby number and compared with the analytical solutions. In particular, we present the temporal evolution of SSD over a flat bottom and slope for a current with $U=0.4 \mathrm{~m} \mathrm{~s}^{-1}, \epsilon=0.4$. We demonstrate the different dynamical roles of momentum advection and buoyancy advection in setting the strength of the vertical circulation. 

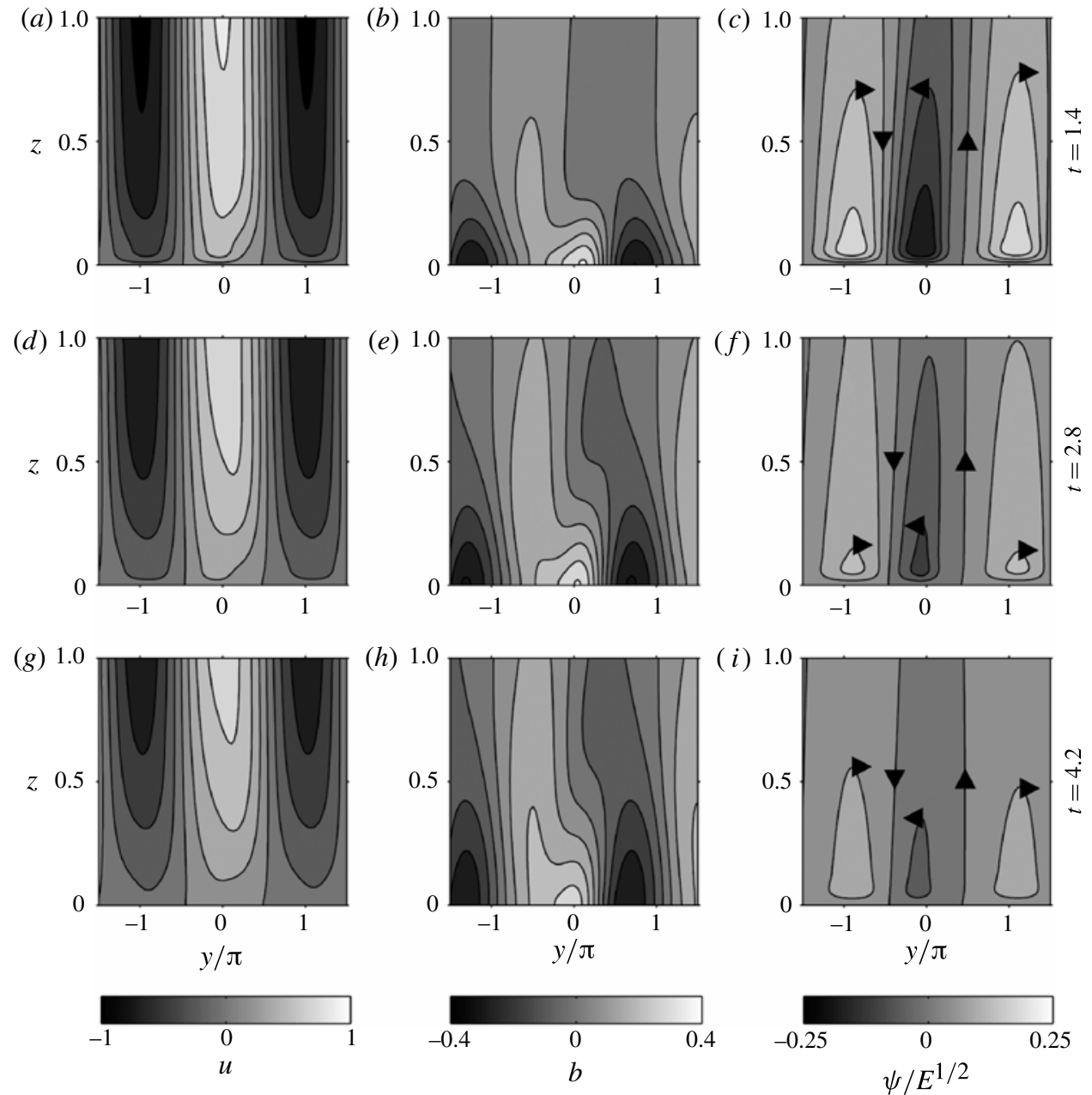

FIgURE 7. $\beta=1, \theta=0.01, \epsilon=0.4$. The stratified spindown ROMS solutions are shown in the rotated coordinate frame at $t=1.4(a-c), 2.8(d-f)$, and $4.2(g-i)$. The along-isobath flow, $u(a, d, g)$, is contoured every 0.2 units, the buoyancy anomaly, $b \quad(b, e, h)$, is contoured every 0.08 units, and the secondary circulation, $\psi / E^{1 / 2}(c, f, i)$, is contoured every 0.05 units.

Over a flat bottom (figure 6), momentum advection modifies stratified spindown and the vertical circulation in two ways. First, the along-isobath flow weakens faster about the cyclonic axis, $y_{c}=\pi / 2$, than the anticyclonic axis, $y_{a}=-\pi / 2$, and is caused by lateral momentum advection. Second, momentum advection causes Ekman pumping to decay faster than Ekman suction. The secondary circulation streamlines spread apart on the cyclonic axis and squeeze together on the anticyclonic axis.

Over a slope, new features emerge as buoyancy advection modifies the spindown dynamics. Figure 7 shows the current's temporal evolution for $\theta=0.01, \beta=1, U=$ $0.4 \mathrm{~m} \mathrm{~s}^{-1}$, and $\epsilon=0.4$. In contrast to the flat bottom configuration, the buoyancy field has its maximum and minimum anomalies within the thermal boundary layer. These buoyancy anomalies tend to converge on the cyclonic axis. The isopycnals (not shown) indicate the formation of a density front, a maximum in the cross- 


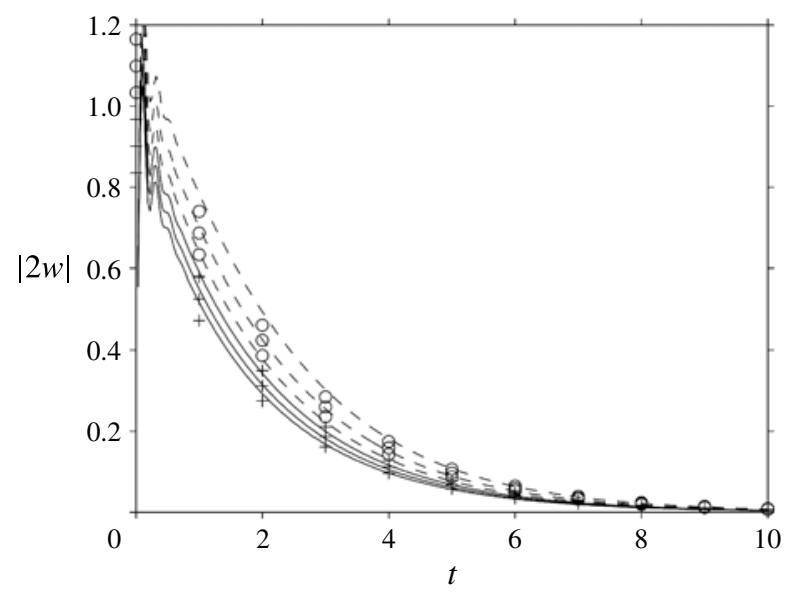

FIGURE 8. For HSD over a flat bottom, the ROMS solutions for Ekman pumping (solid line) at $y_{c}$ and Ekman suction (dashed line) at $y_{a}$ are compared with the analytical solutions for Ekman pumping (+) and Ekman suction $(\bigcirc)$. The solutions are shown for $\epsilon=0.1,0.3,0.5$ with increasing asymmetry for increasing Rossby number.

isobath density gradient, on the cyclonic axis. The buoyancy anomaly convergence correlates with a weakening in the near-bottom geostrophic flow and the cyclonic vorticity. The secondary circulation shows that the streamlines are more widely spaced on the cyclonic axis than on the anticyclonic axis. This spacing is consistent with the predicted behaviour due to nonlinear buoyancy shutdown and Ekman momentum advection. The correction from nonlinear buoyancy shutdown scales as $O\left(\beta^{1 / 2} E^{-1 / 4} \epsilon\right)=6.3 \epsilon$ and is expected to modify the secondary circulation to a greater extent than momentum advection. In contrast to the flat bottom solution, cyclonic vorticity spins down more slowly than anticyclonic vorticity in the interior. This slower spindown is consistent with weaker Ekman pumping rather than Ekman suction, which opposes momentum advection in the interior. Thus, over a slope, nonlinear buoyancy shutdown contributes in weakening Ekman pumping to a greater extent than Ekman suction. The resulting secondary circulation opposes the momentum advection effect on the asymmetry in the interior vertical vorticity.

The numerical model solutions are compared with the analytical solutions to evaluate the relative contributions of momentum advection and buoyancy advection to Ekman pumping and suction. The temporal decay in Ekman pumping at $y_{c}$ and Ekman suction at $y_{a}$ is shown in figure 8 for HSD over a flat bottom and figure 9 for SSD over a slope. From the analytical results, Ekman pumping is $w_{p}=w_{i}\left(y_{c}, z=0\right)$ and Ekman suction is $w_{s}=-w_{i}\left(y_{a}, z=0\right)$. In the numerical model, Ekman pumping and suction are measured by the vertical velocity evaluated at $z=0.08$, outside the Ekman layer base at $z=0.03$. This height is chosen from the average height of the maximum absolute vertical velocity on the cyclonic and anticyclonic axes. During SSD over a slope, Ekman pumping and Ekman suction are influenced by both nonlinear buoyancy shutdown and momentum advection. Despite the presence of momentum advection, the numerical solutions to Ekman pumping and suction by nonlinear buoyancy shutdown show good initial agreement with the analytical prediction. Although the analytical theory for nonlinear buoyancy shutdown is strictly valid for $\epsilon<\beta^{-1 / 2} E^{1 / 4}=0.16$ given the model parameters, the correction is applied to $\epsilon=0.5$. 


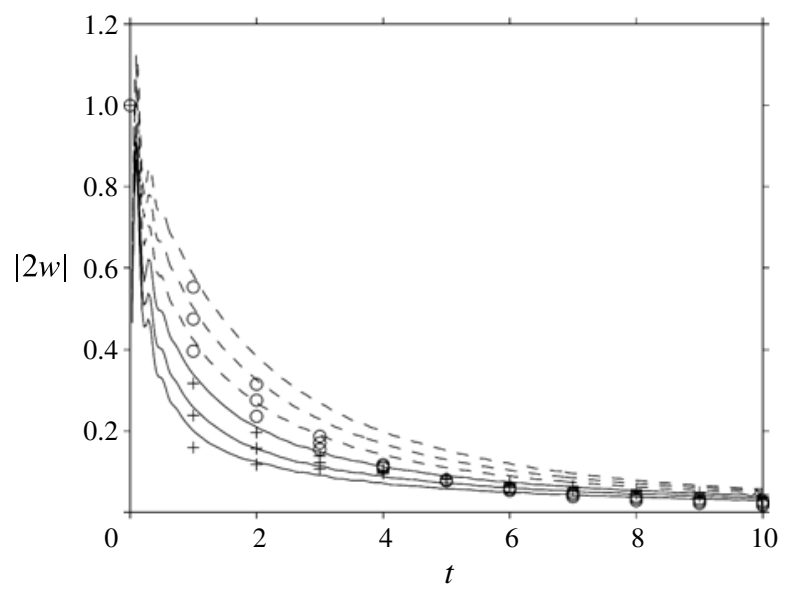

FIGURE 9. For SSD over a slope, with $\beta=1$, the ROMS solutions for Ekman pumping (solid line) at $y_{c}$ and Ekman suction (dashed line) at $y_{a}$ are compared with the analytical solutions for Ekman pumping $(+)$ and Ekman suction $(O)$. The solutions are shown for $\epsilon=0.1,0.3,0.5$ with increasing asymmetry for increasing Rossby number.

The numerical solutions for Ekman pumping and suction show a slower decay rate than the analytical solutions. For HSD over a flat bottom, this difference may arise due to an imperfect implementation of the no-slip boundary condition in the numerical model. The difference is also explained by the $O\left(\epsilon^{2}\right)$ correction providing a non-negligible contribution at higher Rossby number. From Hart (2000)'s formula, the $O\left(\epsilon^{2}\right)$ correction is $15 \epsilon / 56$ as large as the $O(\epsilon)$ correction, ranging from 0.02 for $\epsilon=0.1$ to 0.13 for $\epsilon=0.5$. This $O\left(\epsilon^{2}\right)$ correction tends to enhance both Ekman pumping and suction, which is consistent with the discrepancy between the numerical and analytical solutions.

For SSD over a slope, the slower decay rates may be explained by several reasons, including an imperfect no-slip boundary condition in the numerical model. At early time, $t<1$, nonlinear buoyancy shutdown reduces Ekman pumping and suction in the numerical solutions, although these quantities are systematically larger than the analytical solutions. This systematic error may be explained by an $O\left(E^{1 / 4}\right)$ correction from vertical advection of the laterally uniform buoyancy anomaly in the thermal boundary layer (Benthuysen 2010). This correction causes Ekman pumping and suction to increase from the $O(1)$ solution during SSD over a flat bottom. At later times, $t>1$, the correction from nonlinear buoyancy shutdown in the analytical solutions underestimates the difference between Ekman pumping and suction. The breakdown in the theory is in part due to the neglect of momentum advection. In the analytical theory, vertical diffusion of the buoyancy anomalies weakens the correction to Ekman pumping, leading to a reversal in its sign. In the numerical solutions, Ekman advection of momentum appears to become important in sustaining the asymmetry in Ekman pumping and suction throughout spindown. Despite the discrepancy between the analytical and numerical solutions, the asymmetry in Ekman pumping and suction at early times is consistent with the prediction by nonlinear buoyancy shutdown. This indicates that momentum advection plays a secondary role then.

The ratio of Ekman pumping, $w_{p}$, to Ekman suction, $w_{s}$, is shown in figure 10 at $t=1.4$ for increasing Rossby number. For HSD over a flat bottom and 


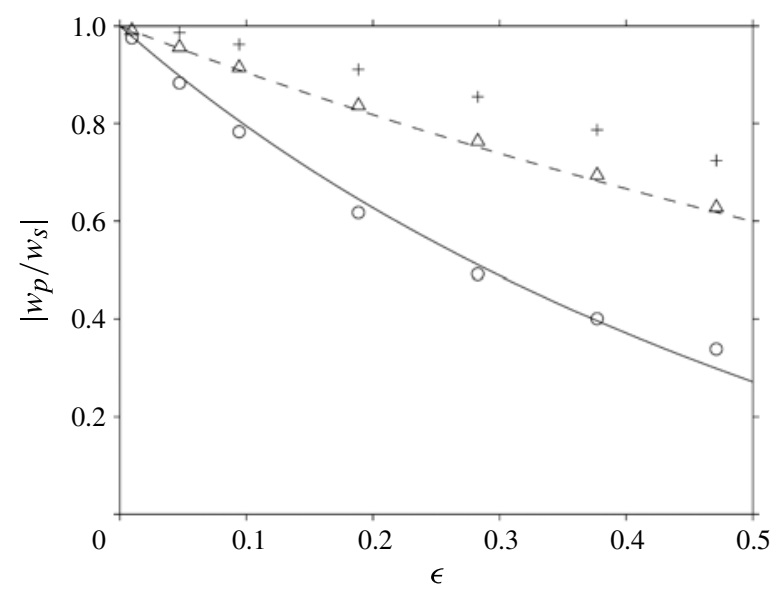

FIGURE 10. The ratio of Ekman pumping, $w_{p}$, to Ekman suction, $w_{s}$, is presented at $t=1.4$ for increasing Rossby number. The ROMS solutions are presented for HSD over a flat bottom $(\triangle)$, SSD over a flat bottom $(+)$, and SSD over a slope $(\bigcirc)$. The analytical solutions are shown for HSD over a flat bottom (dashed line), calculated from (4.66)-(4.67) and SSD over a slope (solid line), calculated from (4.32) with $\beta=1$.

$u(t=0)=\cos (y)$ this ratio is (Benthuysen \& Thomas 2012a)

$$
\left|\frac{w_{p, H S D}}{w_{s, H S D}}\right|=\frac{1-\epsilon \alpha_{1}(t)}{1+\epsilon \alpha_{1}(t)}
$$

where

$$
\alpha_{1}(t)=\frac{13}{20}\left(1-\frac{6}{13} \exp (-t / 2)\right) .
$$

From the above ratio, $\alpha_{1}(t=1.4)=0.50$, and a MacLaurin series expansion in $\epsilon$ yields the ratio $\left|w_{p, H S D} / w_{s, H S D}\right|=1-\epsilon$ for $\epsilon \ll 1$.

For SSD over a slope, with $\beta=1, w_{p}^{(1)} / w_{p}^{(0)}=-0.18$ at $t=1.4$, and a MacLaurin series expansion in $\epsilon$, where $\epsilon \ll 1$, yields the ratio $\left|w_{p} / w_{s}\right|=1-0.36 E^{-1 / 4} \epsilon$ or $1-2.3 \epsilon$. These measures show that the asymmetry in Ekman pumping and suction becomes more pronounced by nonlinear buoyancy shutdown than momentum advection for increasing Rossby number. Although the analytical estimates for Ekman pumping and suction show deviations from the numerical solution, the ratio $\left|w_{p} / w_{s}\right|$ shows agreement. For early times, the ratio of Ekman pumping to Ekman suction for SSD over a flat bottom is weaker than the ratio from HSD. This result occurs because the aforementioned $O\left(E^{1 / 4}\right)$ correction increases both Ekman pumping and suction, thereby reducing the ratio. The significant point in figure 10 is that the asymmetry in Ekman pumping and suction shown for SSD over a slope cannot be accounted for by momentum advection alone.

The asymmetry in Ekman pumping at $y_{c}$ and Ekman suction at $y_{a}$ has consequences for the lateral structure of Ekman pumping. Figure 11 shows the temporal evolution in Ekman pumping for increasing Rossby number. In each configuration, weaker Ekman pumping at $y_{c}$ than Ekman suction at $y_{a}$ is balanced by Ekman pumping where the initial geostrophic flow is an absolute maximum or minimum, i.e. at $y / \pi=m$ and $m=-1,0,1$. The asymmetry in Ekman pumping at $y_{c}$ and Ekman suction at $y_{a}$ becomes more pronounced for increasing Rossby number. In the SSD solutions with 

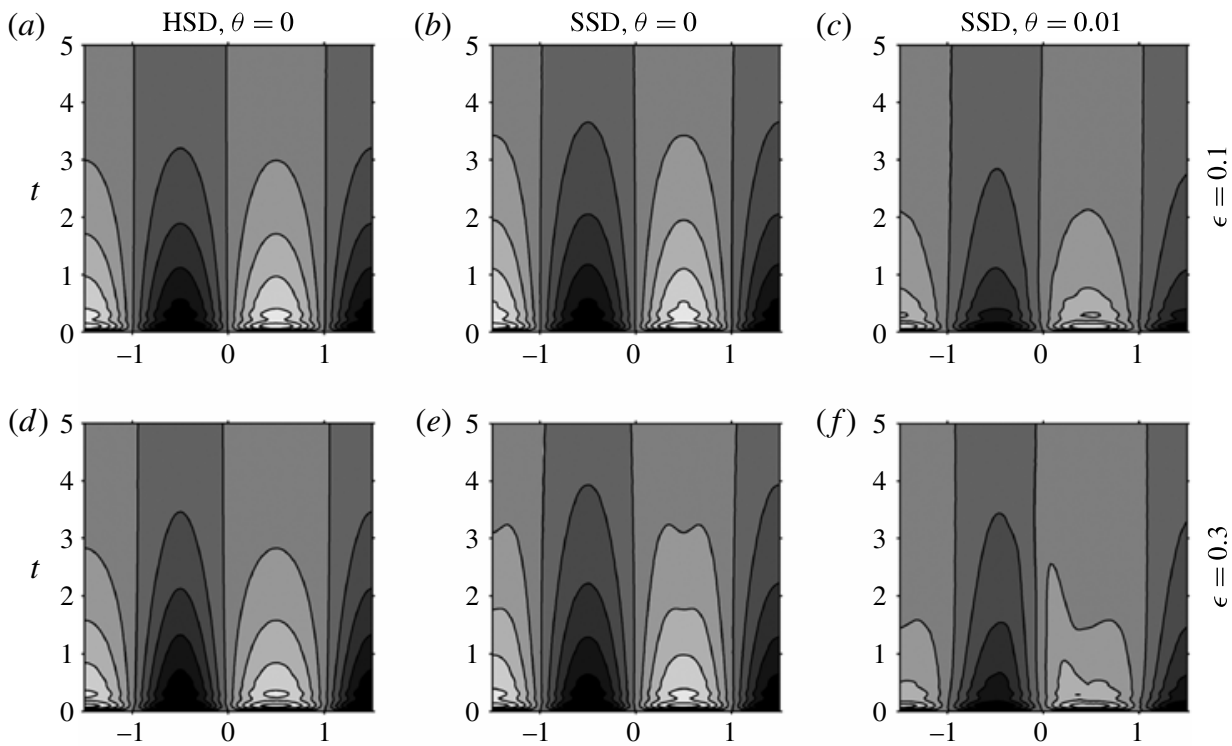

(e)

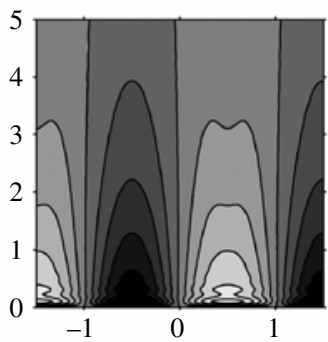

(f) 5
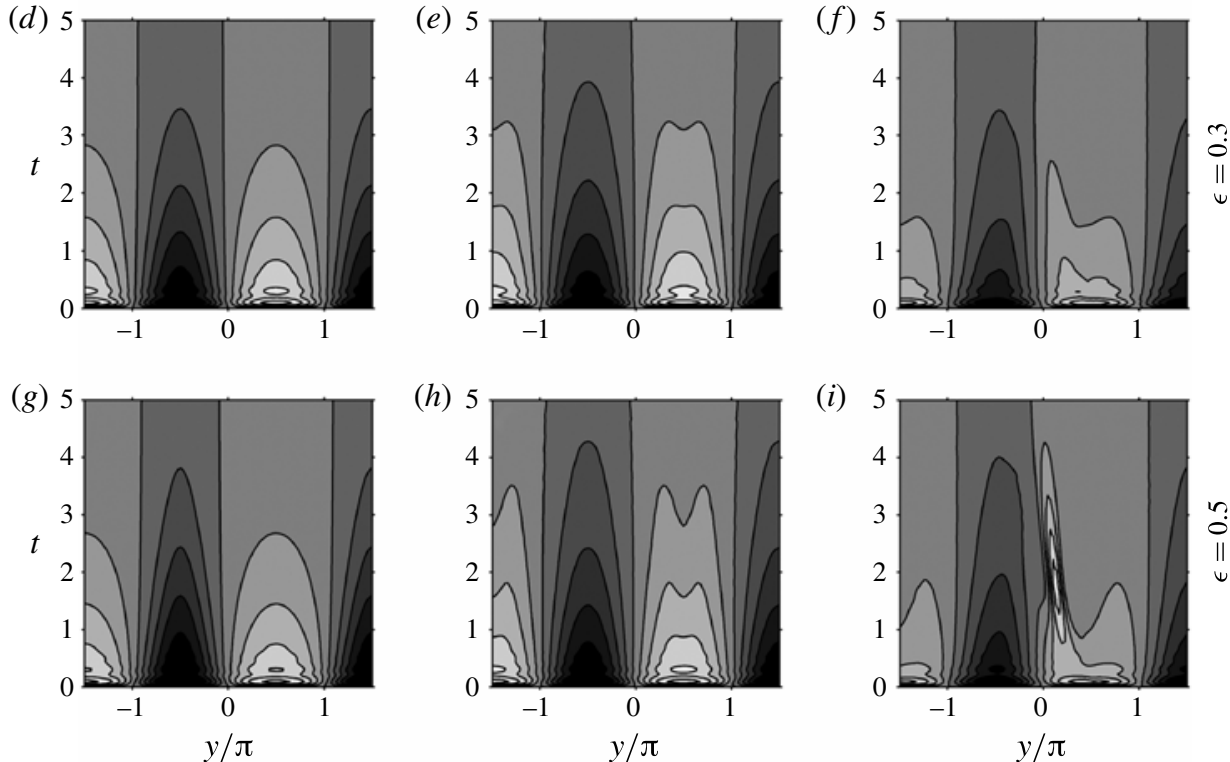

(h)

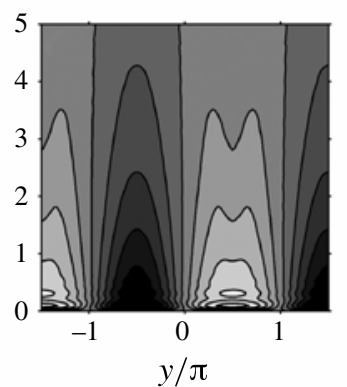

(i)

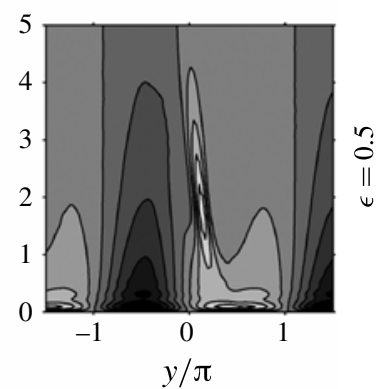

$-1.0$

$-0.5$

$2 w$

FIGURE 11. Ekman pumping is shown for $\epsilon=0.1(a-c), 0.3(d-f), 0.5(g-i)$ and contoured every 0.2 units. The ROMS solutions are shown for HSD over a flat bottom $(a, d, g)$, SSD over a flat bottom $(b, e, h)$ or over a slope $(c, f, i)$.

higher Rossby numbers, Ekman pumping becomes greater off the cyclonic axis than on the cyclonic axis. Interestingly, for SSD over a slope with $\epsilon=0.5$, an intense spike of Ekman pumping occurs at $t=1.71$ and $y / \pi=0.13$ (figure $11 i$ ). The only other time when the Ekman pumping reaches this value is at $t=0.09$, during the initial formation of the Ekman layer. Thus, buoyancy advection is shown to play a significant role in altering Ekman pumping away from the cyclonic axis. Intense vertical flows out of the boundary layer are produced even where the far-field, geostrophic vertical relative vorticity is zero.

\subsection{Asymmetries in spindown of cyclonic and anticyclonic vorticity}

In this section, we consider the asymmetries that arise in the vertical relative vorticity field during spindown. For the $\epsilon=0.4$ numerical solutions, the spindown of the vertical relative vorticity on the cyclonic axis and on the anticyclonic axis is compared in figure 12. The vertical relative vorticity in HSD over a flat bottom (figure 12a) 
(a)
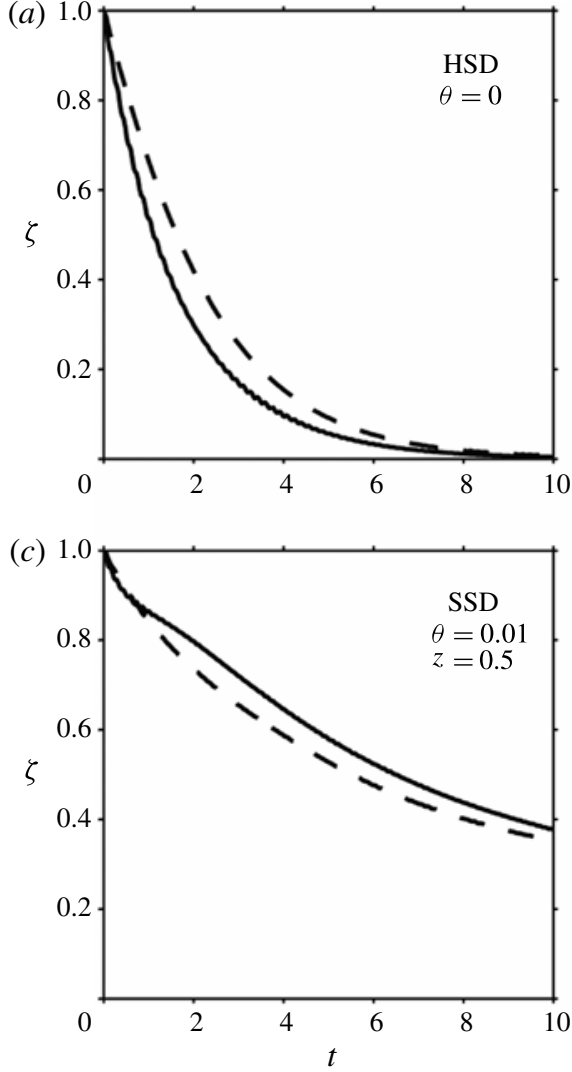

(b)

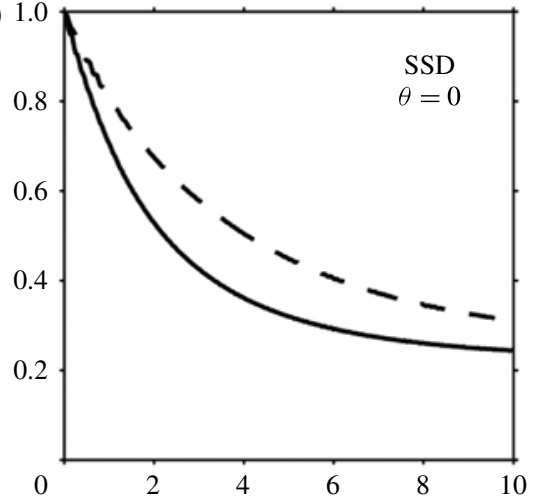

(d)

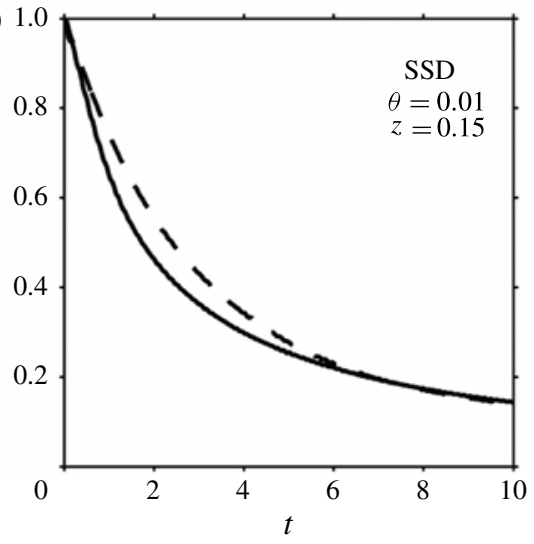

FIGURE 12. Cyclonic vorticity (solid line), evaluated at $y_{c}=\pi / 2$, and anticyclonic vorticity (dashed line), evaluated at $y_{a}=-\pi / 2$, decay asymmetrically for $\epsilon=0.4$. The ROMS solutions are shown for (a) HSD over a flat bottom in the interior domain, (b) SSD over a flat bottom in the interior at $z=0.5$, and SSD over a slope $(c)$ in the interior at $z=0.5$ and $(d)$ in the thermal boundary layer at $z=0.15$.

decays faster than SSD over a flat bottom (figure 12b) because SSD has vertical shear in the geostrophic flow. In both configurations, cyclonic vorticity, $\zeta_{c}$, decays faster than anticyclonic vorticity, $\zeta_{a}$. The ratio $\left|\zeta_{c} / \zeta_{a}\right|$ decreases from 0.76 at $t=1.4$ to 0.69 at $t=2.8$ in figure $12(a)$ and from 0.83 at $t=1.4$ to 0.74 at $t=2.8$ in figure $12(b)$.

Similarly, over a stratified slope, the interior cyclonic vorticity initially decays faster than anticyclonic vorticity at $z=0.5$ (figure $12 c$ ). Within an inertial period, the interior secondary circulation is set-up and spins down the current over a Prandtl depth. During this initial spindown, lateral and vertical momentum advection causes cyclonic vorticity to spin down faster than anticyclonic vorticity. However, in contrast to the flat bottom solutions, cyclonic vorticity becomes greater than anticyclonic vorticity at $t=0.7$ and remains greater. At $z=0.5$, the ratio $\left|\zeta_{c} / \zeta_{a}\right|$ grows from 1.05 at $t=1.4$ to 1.10 at $t=2.8$. In contrast to in the interior, cyclonic vorticity decays faster than anticyclonic vorticity in the thermal boundary layer at $z=0.15$ (figure $12 d$ ). These temporal differences support the notion that nonlinear buoyancy shutdown can oppose momentum advection impact on vertical vorticity during spindown. 
(a)

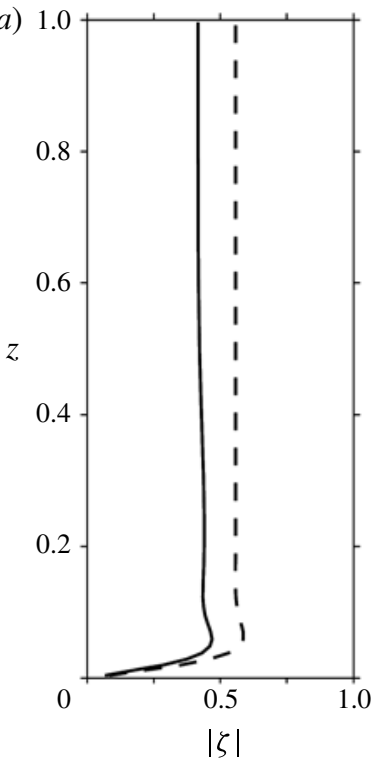

(b)

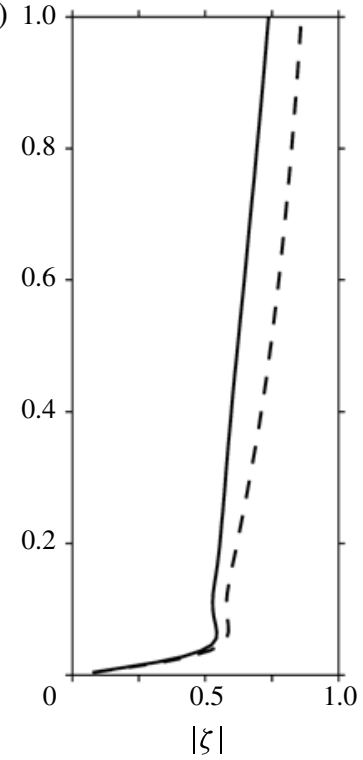

(c)

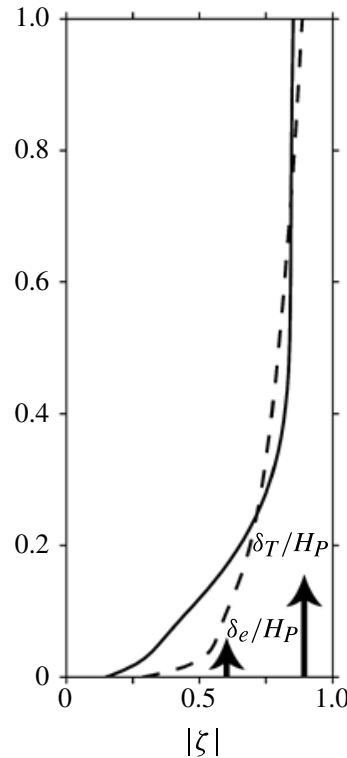

FIGURE 13. Vertical profiles of cyclonic vorticity (solid line), evaluated at $y_{c}=\pi / 2$, and anticyclonic vorticity (dashed line), evaluated at $y_{a}=-\pi / 2$, for $t=1.4$ and $\epsilon=0.4$. The ROMS solutions are shown for $(a)$ HSD over a flat bottom, $(b)$ SSD over a flat bottom, and (c) SSD over a slope. The Ekman and thermal boundary layer depths are indicated in $(c)$.

For each configuration, the vertical profiles of cyclonic and anticyclonic vorticity are shown in figure 13. By $t=1.4$, the mean vertical relative vorticity is reduced to approximately half of its initial value in HSD over a flat bottom. Similarly, for SSD over a flat bottom, cyclonic vorticity is weaker than anticyclonic vorticity outside the Ekman boundary layer. For these solutions, the profiles approach one in the far-field because the secondary circulation tends to spin down the current over a Prandtl depth. For SSD over a slope, the vertical profiles are more complex. At one Prandtl depth, cyclonic vorticity is slightly less than anticyclonic vorticity. However, between $z=0.25$ and $z=0.73$, cyclonic vorticity is greater than anticyclonic vorticity. In this region, the interior secondary circulation spins down anticyclonic vorticity faster. This occurs because Ekman suction is greater than Ekman pumping to the extent that it opposes the effects of momentum advection. Within the thermal boundary layer, cyclonic vorticity is notably weaker than anticyclonic vorticity and is correlated with buoyancy anomalies converging on the cyclonic axis.

The ratio of cyclonic vorticity to anticyclonic vorticity is shown in figure 14 for increasing Rossby number. For nonlinear homogeneous spindown, this ratio is (Benthuysen \& Thomas 2012a)

$$
\left|\frac{\zeta_{c, H S D}}{\zeta_{a, H S D}}\right|=\frac{1-\epsilon \alpha_{2}(t)}{1+\epsilon \alpha_{2}(t)}
$$

where

$$
\alpha_{2}(t)=\frac{13}{20}(1-\exp (-t / 2))
$$




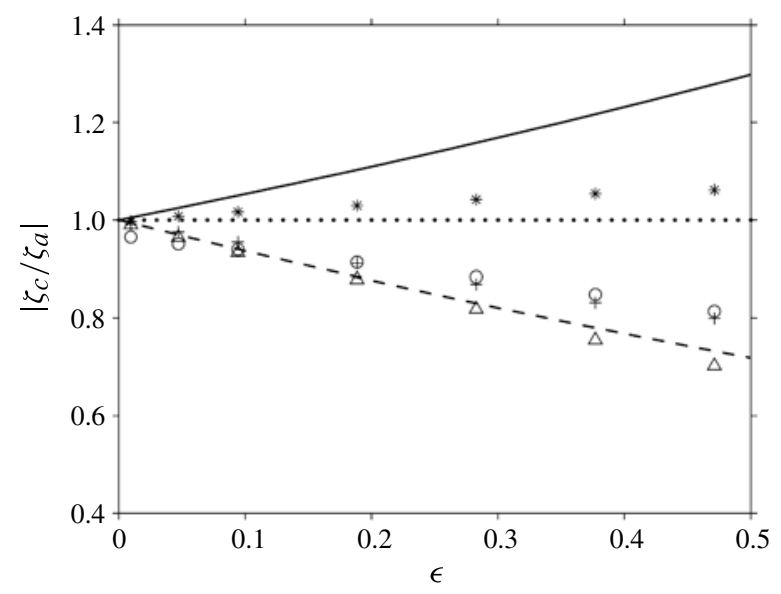

FIGURE 14. The ratio of cyclonic vorticity, $\zeta_{c}$, at $y_{c}$ to anticyclonic vorticity, $\zeta_{a}$, at $y_{a}$ is presented at $t=1.4$ for increasing Rossby number. The ROMS solutions are shown for HSD over a flat bottom $(\triangle)$ at $z=0.5$, SSD over a flat bottom at $z=0.5(+)$, and SSD over a slope at $z=0.15(\bigcirc)$ and at $z=0.5(*)$. The analytical solutions are shown for HSD over a flat bottom at $z=0.5$ (dashed line), evaluated from (4.64)-(4.65), and SSD over a slope at $z=0.5$ (solid line), where $\beta=1$ is applied. The axis $\left|\zeta_{c} / \zeta_{a}\right|=1$ is indicated by the dotted line.

At $t=1.4$, the flat bottom solutions at $z=0.5$ and the slope solution at $z=0.15$ show that cyclonic vorticity is increasingly weaker than anticyclonic vorticity for increasing Rossby number. The ratio for the HSD analytical solution (5.3) agrees with the numerical solutions.

For SSD over a slope, the numerical solutions at $z=0.5$ show that the ratio of cyclonic to anticyclonic vorticity increases for increasing Rossby number. The analytical ratios calculated using (4.68)-(4.69) overestimate the numerical solution ratios. Thus, at $t=1.4$, nonlinear buoyancy shutdown alone overestimates the asymmetry between cyclonic and anticyclonic vorticity. This overestimate occurs because momentum advection is neglected in the nonlinear buoyancy shutdown solution. However, nonlinear buoyancy shutdown is still necessary to explain the asymmetry in cyclonic and anticyclonic vorticity for SSD over a slope.

\section{Conclusions}

For stratified spindown over a slope, new criteria have been identified for when buoyancy advection is important in generating asymmetries in vertical velocity and vertical relative vorticity. Cross-isobath Ekman advection of buoyancy and vertical advection of buoyancy strengthens (weakens) buoyancy anomalies within the thermal boundary layer about the cyclonic (anticyclonic) axis. From geostrophy, the cyclonic vorticity is weakened to a greater extent than anticyclonic vorticity in the thermal boundary layer. This process, defined here as nonlinear buoyancy shutdown, weakens Ekman pumping more than Ekman suction at $O\left(\beta^{1 / 2} E^{-1 / 4} \epsilon\right)$ and can enter into the leading-order dynamics when $\beta^{1 / 2} E^{-1 / 4} \epsilon>1$.

Nonlinear buoyancy shutdown's suppression of Ekman pumping more than Ekman suction has consequences for the interior vertical vorticity's evolution. If buoyancy advection modifies the secondary circulation to a greater extent than $O(\epsilon)$, then the effects of momentum advection are subdominant. Hence, the interior anticyclonic 
vorticity decays faster than cyclonic vorticity. This asymmetric decay contrasts with nonlinear spindown over a flat bottom in either a stratified or homogeneous fluid. In these configurations, the interior cyclonic vorticity decays faster than anticyclonic vorticity. Numerical solutions support the predictions of the asymmetries in the vertical circulation and vertical relative vorticity due to nonlinear buoyancy shutdown.

Nonlinear buoyancy shutdown may play an important role in setting the lateral structure of slope currents over topography for flows with $\beta>1$. At mid-latitudes $\left(f=10^{-4} \mathrm{~s}^{-1}\right)$, we estimate $\beta$ for typical continental shelves $\left(N^{2}=10^{-5}-10^{-4} \mathrm{~s}^{-2}, \theta=\right.$ $\left.10^{-4}-10^{-3}\right)$ and continental slopes $(\theta=0.005-0.01)$. For an Ekman depth of $\delta_{e}=10 \mathrm{~m}$ and a water depth of $O(100 \mathrm{~m})$, the Ekman number is $E=0.01$ and yields values of $\beta=10^{-9}-10^{-3}$ over the continental shelf and $\beta=0.01-10$ over the continental slope. This range of values suggests that buoyancy shutdown may occur to a greater extent over the continental slope than over the continental shelf.

On the lower continental slope, measurements of the North Atlantic Deep Western Boundary Current at the Blake Outer Ridge reveal a frictional bottom boundary layer embedded within a thicker bottom mixed layer (Stahr \& Sanford 1999). The bottom mixed layer is thicker and the Ekman transport is weaker on the downslope side of the current (see figures 11 and 12 in Stahr \& Sanford 1999). This feature is also a characteristic of nonlinear buoyancy shutdown (e.g. figure $2 b$ ) and thus may be evidence of this process. Having said this, the cross-isobath density gradients within the bottom mixed layer in the observations are small with little vertical shear in the along-slope flow, indicating that other processes govern the dynamics than linear and nonlinear buoyancy shutdown.

In summary, this work has provided insight into the nonlinear coupling between frictionally driven flows and the buoyancy field. Future challenges include identifying features of nonlinear buoyancy shutdown in observations and understanding how this mechanism can modify the evolution of three-dimensional structures, such as slope vortices, and their vertical circulation.

\section{Appendix. Discretization of the equations for linear and nonlinear stratified spindown}

This appendix presents the numerical method that is used to solve for the $n=0,1$ corrections to stratified spindown on a sloping bottom to validate the $n=0$ analytical solution and ROMS solutions. The equations are solved using the Crank-Nicolson method (Crank \& Nicolson 1947), in which the partial derivatives in time and space are discretized at a fictitious point in between two time steps.

The labels $j$ and $k$ denote the non-dimensional time index and the thermal boundary layer vertical coordinate index, respectively. The numerical solution is calculated on a grid from $1 \leqslant j \leqslant J$ and $1 \leqslant k \leqslant K$, where the initial conditions are applied at $j=1$ and the maximum number of time steps and spatial steps in the domain are given by $J$ and $K$, respectively. The linear problem is solved by first discretizing the set of equations, boundary conditions, and initial conditions (4.37)-(4.45) that describe the linear dynamics of spindown and buoyancy shutdown. Then, the numerical solution for the linear dynamics is applied to the set of equations and boundary conditions that describe nonlinear buoyancy shutdown and (4.56)-(4.60). 
A.1. Discretization of the $n=0$ equations

At $n=0$, the interior along-isobath flow is $U_{i}^{(0)}(t, z)=1-\Psi^{(0)}(t) \exp (-z)$. The interior along-isobath flow evolves as

$$
\frac{\mathrm{d} \Psi^{(0)}}{\mathrm{d} t}+\varphi^{(0)}=0
$$

where $\varphi^{(0)}$ is solved below. The coupled set of partial differential equations reduces to the diffusion equation

$$
\frac{\partial U_{T}^{(0)}}{\partial t}=\frac{1}{2} \frac{\partial^{2} U_{T}^{(0)}}{\partial \xi^{2}},
$$

for $t>0,0<\xi<\infty$, subject to the following boundary conditions of no-slip, Ekman pumping, no-normal-buoyancy-flux at the bottom, and no far-field thermal boundary layer flow:

$$
\begin{gathered}
\varphi^{(0)}+\frac{1}{2}\left(1-\Psi^{(0)}+\beta^{1 / 2} U_{T}^{(0)}(\xi=0)\right)=0, \\
\frac{\mathrm{d} \varphi^{(0)}}{\mathrm{d} t}+\frac{1}{2}\left(\varphi^{(0)}+\beta^{1 / 2} \frac{\partial U_{T}^{(0)}}{\partial t}(\xi=0)\right)=0, \\
\frac{\partial U_{T}^{(0)}}{\partial \xi}(\xi=0)+2 \varphi^{(0)}=0, \\
U_{T}^{(0)} \rightarrow 0 \quad \text { as } \xi \rightarrow \infty
\end{gathered}
$$

and the following initial conditions:

$$
\left(\Psi^{(0)}(t=0), U_{T}^{(0)}(t=0), \varphi^{(0)}(t=0)\right)=\left(0,0,-\frac{1}{2}\right) .
$$

These equations are discretized, where $\lambda \equiv 1 / 2$ for the Crank-Nicolson scheme, $q \equiv \Delta t / \Delta \xi, r \equiv \Delta t /\left(2(\Delta \xi)^{2}\right), \Delta t$ is the non-dimensional time step, and $\Delta \xi$ is the non-dimensional thermal boundary layer grid step. The discretized diffusion equation is

$$
\begin{aligned}
& -\left.\lambda r U_{T}^{(0)}\right|_{j+1} ^{k+1}+\left.(1+2 \lambda r) U_{T}^{(0)}\right|_{j+1} ^{k}-\left.\lambda r U_{T}^{(0)}\right|_{j+1} ^{k-1} \\
& =\left.(1-\lambda) r U_{T}^{(0)}\right|_{j} ^{k+1}+\left.(1-2 r(1-\lambda)) U_{T}^{(0)}\right|_{j} ^{k}+\left.(1-\lambda) r U_{T}^{(0)}\right|_{j} ^{k-1},
\end{aligned}
$$

for $j>1,1<k<K$. The boundary condition for $U_{T}^{(0)}$ at $\xi=0$ is derived such that it is consistent with the diffusion equation, where

$$
\left.\frac{\partial U_{T}^{(0)}}{\partial t}\right|_{j+\frac{1}{2}} ^{k=1}=\left.\left(\frac{\lambda}{2}\right) \frac{\partial^{2} U_{T}^{(0)}}{\partial \xi^{2}}\right|_{j+1} ^{k=1}+\left.\left(\frac{1-\lambda}{2}\right) \frac{\partial^{2} U_{T}^{(0)}}{\partial \xi^{2}}\right|_{j} ^{k=1}
$$

From an expansion of $U_{T}^{(0)}$ at $k$,

$$
\left.\frac{\partial^{2} U_{T}^{(0)}}{\partial \xi^{2}}\right|_{j} ^{k}=\frac{2}{(\Delta \xi)^{2}}\left(\left.U_{T}^{(0)}\right|_{j} ^{k+1}-\left.U_{T}^{(0)}\right|_{j} ^{k}\right)-\left.\left(\frac{2}{\Delta \xi}\right) \frac{\partial U_{T}^{(0)}}{\partial \xi}\right|_{j} ^{k}
$$


Then, at $k=1$, with (A 5),

$$
\left.\frac{\partial^{2} U_{T}^{(0)}}{\partial \xi^{2}}\right|_{j} ^{k=1}=\frac{2}{(\Delta \xi)^{2}}\left(\left.U_{T}^{(0)}\right|_{j} ^{k=2}-\left.U_{T}^{(0)}\right|_{j} ^{k=1}\right)+\left.\left(\frac{4}{\Delta \xi}\right) \varphi^{(0)}\right|_{j} .
$$

Equation (A 4) is discretized on the Crank-Nicolson stencil at $j+1 / 2, k=1$, so that

$$
\left.\varphi^{(0)}\right|_{j+1}=\left.\left(\frac{4-\Delta t}{4+\Delta t}\right) \varphi^{(0)}\right|_{j}-\left(\frac{2 \beta^{1 / 2}}{4+\Delta t}\right)\left(\left.U_{T}^{(0)}\right|_{j+1} ^{k=1}-\left.U_{T}^{(0)}\right|_{j} ^{k=1}\right) .
$$

Then, from substituting (A 11)-(A 12) into (A 9), the boundary condition for $U_{T}^{(0)}, j>$ 1 , becomes

$$
\begin{aligned}
(1+ & \left.2 \lambda r+\frac{4 \beta^{1 / 2} \lambda q}{4+\Delta t}\right)\left.U_{T}^{(0)}\right|_{j} ^{k=1}-\left.2 \lambda r U_{T}^{(0)}\right|_{j} ^{k=2} \\
= & \left.\left(1+\frac{4 \beta^{1 / 2} \lambda q}{4+\Delta t}-2(1-\lambda) r\right) U_{T}^{(0)}\right|_{j-1} ^{k=1}+\left.2(1-\lambda) r U_{T}^{(0)}\right|_{j-1} ^{k=2} \\
& +\left.\left(2 \lambda q\left(\frac{4-\Delta t}{4+\Delta t}\right)+2(1-\lambda) q\right) \varphi^{(0)}\right|_{j-1} .
\end{aligned}
$$

The boundary condition as $\xi \rightarrow \infty$ becomes

$$
\left.U_{T}^{(0)}\right|_{j} ^{k=K}=0
$$

The initial conditions become

$$
\left(\left.\Psi^{(0)}\right|_{j=1},\left.U_{T}^{(0)}\right|_{j=1} ^{k},\left.\varphi^{(0)}\right|_{j=1}\right)=\left(0,0,-\frac{1}{2}\right) .
$$

From (A 8), $U_{T}^{(0)}$ is determined at each time step, $j>1$, by solving

$$
\left.\boldsymbol{A}_{0} \boldsymbol{U}_{T}^{(0)}\right|_{j} ^{1 \leqslant k \leqslant K-1}=\boldsymbol{b}_{0}
$$

where $\boldsymbol{A}_{0}$ is a $K-1 \times K-1$ tridiagonal coefficient matrix with elements determined by the left-hand side of (A 8), $\left.\boldsymbol{U}_{T}^{(0)}\right|_{j} ^{1 \leqslant k \leqslant K-1}$ is the $K-1 \times 1$ solution column vector, and $\boldsymbol{b}_{0}$ is the $K-1 \times 1$ column vector with elements determined by the right-hand side of (A 8), subject to the boundary conditions (A 13)-(A 14). Then, once the solution for $U_{T}^{(0)}$ is solved at each time step, $\varphi^{(0)}$ is determined from (A 12). Finally, the time evolution for the interior along-isobath flow is solved from the discretized no-slip boundary condition, (A 3), such that

$$
\left.\Psi^{(0)}\right|_{j}=1+\left.2 \varphi^{(0)}\right|_{j}+\left.\beta^{1 / 2} U_{T}^{(0)}\right|_{j} ^{k=1} .
$$

This set of equations is solved with the Thomas algorithm for $\sigma=1$ and $\beta=1$, where $\Delta t=0.001, \Delta \xi=0.02$ on a grid from $t=0$ to 20 and $\xi=0$ to 10 . Thus, $J=20001, K=501, r=1.25$, and $q=0.05$. The maximum error between the numerical solution for $\varphi^{(0)}$ as calculated above and the analytical solution, (4.51), is $0.02 \%$.

\section{A.2. Discretization of the $n=1$ equations}

At $n=1$, the interior along-isobath flow is $\Psi^{(1)}(t) \equiv U_{i}^{(1)}(t)$. It evolves as

$$
\frac{\mathrm{d} \Psi^{(1)}}{\mathrm{d} t}-2 \varphi^{(1)}=0
$$


and the forced diffusion equation is

$$
\frac{\partial U_{T}^{(1)}}{\partial t}-\frac{1}{2} \frac{\partial^{2} U_{T}^{(1)}}{\partial \xi^{2}}=\frac{1}{2} \varphi^{(0)} \frac{\partial U_{T}^{(0)}}{\partial \xi},
$$

for $t>0,0<\xi<\infty$, subject to the following boundary conditions of no-slip, Ekman pumping, no-normal-buoyancy-flux at the bottom, and no far-field thermal boundary layer flow:

$$
\begin{gathered}
\varphi^{(1)}+\frac{1}{2}\left(\Psi^{(1)}+\beta^{1 / 2} U_{T}^{(1)}(\xi=0)\right)=0, \\
\frac{\mathrm{d} \varphi^{(1)}}{\mathrm{d} t}+\varphi^{(1)}+\left(\frac{\beta^{1 / 2}}{2}\right) \frac{\partial U_{T}^{(1)}}{\partial t}(\xi=0)=0, \\
\frac{\partial U_{T}^{(1)}}{\partial \xi}(\xi=0)+2 \varphi^{(1)}=\varphi^{(0)} U_{T}^{(0)}(\xi=0), \\
U_{T}^{(1)} \rightarrow 0 \quad \text { as } \xi \rightarrow \infty,
\end{gathered}
$$

and the following initial conditions:

$$
\left(\Psi^{(1)}(t=0), U_{T}^{(1)}(t=0), \varphi^{(1)}(t=0)\right)=(0,0,0) .
$$

The forced diffusion equation is discretized using the Crank-Nicolson scheme, where $\lambda \equiv 1 / 2$ and

$$
\begin{aligned}
& -\left.\lambda r U_{T}^{(1)}\right|_{j} ^{k+1}+\left.(1+2 \lambda r) U_{T}^{(1)}\right|_{j} ^{k}-\left.\lambda r U_{T}^{(1)}\right|_{j} ^{k-1} \\
& =\left.(1-\lambda) r U_{T}^{(1)}\right|_{j-1} ^{k+1}+\left.(1-2 r(1-\lambda)) U_{T}^{(1)}\right|_{j-1} ^{k}+\left.(1-\lambda) r U_{T}^{(1)}\right|_{j-1} ^{k-1} \\
& \quad+\frac{q}{16}\left(\left.\varphi^{(0)}\right|_{j}+\left.\varphi^{(0)}\right|_{j-1}\right)\left(\left.U_{T}^{(0)}\right|_{j-1} ^{k+1}-\left.U_{T}^{(0)}\right|_{j-1} ^{k-1}+\left.U_{T}^{(0)}\right|_{j} ^{k+1}-\left.U_{T}^{(0)}\right|_{j} ^{k-1}\right),
\end{aligned}
$$

for $j>1,1<k<K$. The boundary conditions are discretized as in the previous section such that they are consistent with the diffusion equation. Then, at $k=1$, with (A 22),

$$
\begin{aligned}
\left.\frac{\partial^{2} U_{T}^{(1)}}{\partial \xi^{2}}\right|_{j} ^{k=1}= & \frac{2}{(\Delta \xi)^{2}}\left(\left.U_{T}^{(1)}\right|_{j} ^{k=2}-\left.U_{T}^{(1)}\right|_{j} ^{k=1}\right) \\
& +\left.\left(\frac{4}{\Delta \xi}\right) \varphi^{(1)}\right|_{j}-\left.\left.\left(\frac{2}{\Delta \xi}\right) \varphi^{(0)}\right|_{j} U_{T}^{(0)}\right|_{j} ^{k=1} .
\end{aligned}
$$

Equation (A 21) is discretized on the Crank-Nicolson stencil at $j+1 / 2, k=1$, so that

$$
\left.\varphi^{(1)}\right|_{j+1}=\left.\left(\frac{2-\Delta t}{2+\Delta t}\right) \varphi^{(1)}\right|_{j}-\frac{\beta^{1 / 2}}{2+\Delta t}\left(\left.U_{T}^{(1)}\right|_{j+1} ^{k=1}-\left.U_{T}^{(1)}\right|_{j} ^{k=1}\right) .
$$

By substitution of (A 26)-(A 27) into the diffusion equation on the stencil used in the Crank-Nicolson scheme, the boundary condition for $j>1$ becomes

$$
\begin{aligned}
& \left.\left(1+2 \lambda r+\frac{2 \beta^{1 / 2} \lambda q}{2+\Delta t}\right) U_{T}^{(1)}\right|_{j} ^{k=1}-\left.2 \lambda r U_{T}^{(1)}\right|_{j} ^{k=2} \\
& =\left.\left(1+\frac{2 \beta^{1 / 2} \lambda q}{2+\Delta t}-2(1-\lambda) r\right) U_{T}^{(1)}\right|_{j-1} ^{k=1}+\left.2(1-\lambda) r U_{T}^{(1)}\right|_{j-1} ^{k=2}
\end{aligned}
$$




$$
\begin{aligned}
& +\left.\left(2 \lambda q\left(\frac{2-\Delta t}{2+\Delta t}\right)+2(1-\lambda) q\right) \varphi^{(1)}\right|_{j-1}-\left.\left.\lambda q \varphi^{(0)}\right|_{j} U_{T}^{(0)}\right|_{j} ^{k=1} \\
& -\left.\left.(1-\lambda) q \varphi^{(0)}\right|_{j-1} U_{T}^{(0)}\right|_{j-1} ^{k=1}-\left(\frac{\Delta t}{4}\right)\left(\left.\varphi^{(0)}\right|_{j-1}+\left.\varphi^{(0)}\right|_{j}\right)^{2} .
\end{aligned}
$$

The boundary condition as $\xi \rightarrow \infty$ becomes

$$
\left.U_{T}^{(1)}\right|_{j} ^{k=K}=0 .
$$

The initial conditions become

$$
\left(\left.\Psi^{(1)}\right|_{j=1},\left.U_{T}^{(1)}\right|_{j=1} ^{k},\left.\varphi^{(1)}\right|_{j=1}\right)=(0,0,0) .
$$

From (A 25), $U_{T}^{(0)}$ is determined at each time step, $j>1$, by solving

$$
\boldsymbol{A}_{1} \boldsymbol{U}_{T}^{(1)}(j, 1 \leqslant k \leqslant K-1)=\boldsymbol{b}_{1},
$$

where $\boldsymbol{A}_{1}$ is a $K-1 \times K-1$ tridiagonal coefficient matrix with elements determined by the left-hand side of (A 25), $\left.\boldsymbol{U}_{T}^{(1)}\right|_{j} ^{1 \leqslant k \leqslant K-1}$ is the $K-1 \times 1$ solution column vector, and $\boldsymbol{b}_{1}$ is a $K-1 \times 1$ column vector with elements determined by the right-hand side of (A 25), subject to the boundary conditions (A 28)-(A 29). Then, $\varphi^{(1)}$ is determined from (A 27), after the solution for $U_{T}^{(1)}$ is solved at each time step. Finally, the time evolution for the interior along-isobath flow is solved from the discretized no-slip boundary condition, (A 20), such that

$$
\left.\Psi^{(1)}\right|_{j}=-\left.2 \varphi^{(1)}\right|_{j}-\left.\beta^{1 / 2} U_{T}^{(1)}\right|_{j} ^{k=1} .
$$

The above set of equations is solved with the Thomas algorithm, subject to the previously calculated numerical solution for $U_{T}^{(0)}$ and $\varphi^{(0)}$ with $\sigma=1$ and $\beta=1$. The grid is set up from $t=0$ to 10 and $\xi=0$ to 10 , where $\Delta t=0.001, \Delta \xi=0.02, J=$ $10001, K=501, r=1.25$ and $q=0.05$.

\section{REFERENCES}

Abramowitz, M. \& Stegun, I. A. 1972 Handbook of Mathematical Functions. Dover.

BENTHUYSEN, J. A. 2010 Linear and nonlinear stratified spindown over sloping topography. PhD thesis, Massachusetts Institute of Technology and Woods Hole Oceanographic Institution.

Benthuysen, J. A. \& Thomas, L. N. $2012 a$ Asymmetries in vertical vorticity and vertical velocity arising during nonlinear homogeneous spindown. Phys. Fluids 24, 076601.

Benthuysen, J. A. \& Thomas, L. N. $2012 b$ Frictional and diapycnal mixing at a slope: boundary control of potential vorticity. J. Phys. Oceanogr. 42, 1509-1523.

Benton, G. S., Lipps, F. B. \& TUANn, S.-Y. 1964 The structure of the Ekman layer for geostrophic flows with lateral shear. Tellus XVI, 186-199.

Chapman, D. C. 2002 Deceleration of a finite-width, stratified current over a sloping bottom: frictional spindown or buoyancy shutdown? J. Phys. Oceanogr. 32, 336-352.

Charney, J. G. \& Eliassen, A. 1949 A numerical method of predicting the perturbations of the middle-latitude westerlies. Tellus 1, 38-54.

CRANK, J. \& NiCOLSON, P. 1947 A practical method for numerical evaluation of solutions of partial differential equations of the heat-conduction type. Proc. Camb. Phil. Soc. 43, 50-67.

HART, J. E. 1995 Nonlinear Ekman suction and ageostrophic effects in rapidly rotating flows. Geophys. Astrophys. Fluid Dyn. 79, 201-222.

HART, J. E. 2000 A note on nonlinear corrections to the Ekman layer pumping velocity. Phys. Fluids 12, 131-135. 
Holton, J. R. 1965 The influence of viscous boundary layers on transient motions in a stratified rotating fluid: Part I. J. Atmos. Sci. 22, 402-411.

MacCReAdy, P. \& Rhines, P. B. 1991 Buoyant inhibition of Ekman transport on a slope and its effect on stratified spin-up. J. Fluid Mech. 223, 631-661.

Pedlosky, J. 2008 On the weakly nonlinear Ekman layer: thickness and flux. J. Phys. Oceanogr. 38, 1334-1339.

ShchepetKin, A. F. \& MCWilliams, J. C. 2005 The regional oceanic modeling system (ROMS): a split-explicit, free-surface, topography-following-coordinate oceanic model. Ocean Model. 9, 347-404.

STAHR, F. R. \& SANFORD, T. B. 1999 Transport and bottom boundary layer observations of the North Atlantic Deep Western Boundary Current at the Blake Outer Ridge. Deep-Sea Res. II 46, 205-243.

Thomas, L. N. \& Rhines, P. B. 2002 Nonlinear stratified spin-up. J. Fluid Mech. 473, 211-244.

Thorpe, S. A. 1987 Current and temperature variability on the continental slope. Phil. Trans. R. Soc. Lond. A 323, 471-517.

ZaVAla SAnsón, L. 2001 The asymmetric Ekman decay of cyclonic and anticyclonic vortices. Eur. J. Mech. (B/Fluids) 20, 541-556.

Zavala SAnsón, L. \& VAn Heijst, G. J. F. 2000 Nonlinear Ekman effects in rotating barotropic flows. J. Fluid Mech. 412, 75-91. 